\title{
EFFECTS OF LESIONS TO THE DORSAL AND VENTRAL HIPPOCAMPUS ON DEFENSIVE BEHAVIORS IN RATS.
}

\author{
A THESIS SUBMITTED TO THE GRADUATE DIVISION OF THE \\ UNIVERSITY OF HAWAI'I IN PARTIAL FULFILLMENT \\ OF THE REQUIREMENTS FOR THE DEGREE OF \\ MASTER OF ARTS \\ IN \\ PSYCHOLOGY
}

DECEMBER 2004

By

Nathan S. Pentkowski

Thesis Committee:

Robert J. Blanchard, Chairperson

Lorey Takahashi

D. Caroline Blanchard 


\begin{abstract}
This study investigated the role of the dorsal and ventral hippocampus in both unconditioned and conditioned defensive behaviors by examining the effects of pretraining ibotenic acid lesions to the dorsal or ventral hippocampus in Long Evans hooded male rats exposed to three types of threat stimuli, cat odor, a live cat and footshock. Conditioned defensive behaviors were assessed in the same context 24 hours after the threat exposure. During unconditioned test trials, $\mathrm{DH}$ lesions did not significantly alter behaviors during exposure to cat odor, a live cat, or footshock. Additionally, DH lesions failed to modify conditioned behaviors during all three tests of defense. In contrast, VH lesions significantly reduced both unconditioned and conditioned defensive behaviors during cat odor and footshock, while only reducing defensive behaviors during the unconditioned cat exposure trail. These results suggest a role for the $\mathrm{VH}$ in modulating anxiety-like behaviors in certain animal models of defense.
\end{abstract}




\section{TABLE OF CONTENTS}

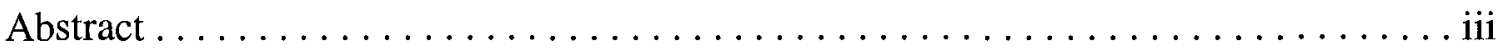

List of Tables ....................................... vii

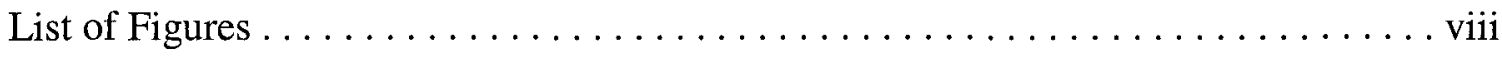

Chapter 1: Introduction $\ldots \ldots \ldots \ldots \ldots \ldots \ldots \ldots \ldots \ldots \ldots \ldots \ldots \ldots \ldots \ldots$

Unconditioned Defensive Behavior ........................ 1

Dorsal and Ventral Hippocampal Specificity .............. 5

Conditioned Defensive Behavior ......................... 7

Chapter 2: Methods and Procedures: Dorsal Hippocampus ............. 10

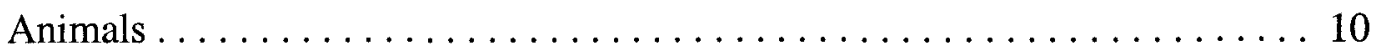

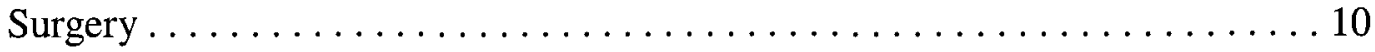

Behavioral Testing Procedures $\ldots \ldots \ldots \ldots \ldots \ldots \ldots \ldots \ldots \ldots \ldots \ldots$

Behavioral Measures ................................ 11

Behavioral Tests ................................. 12

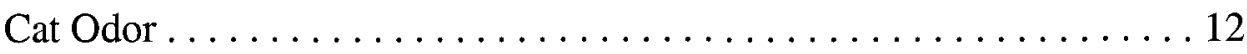

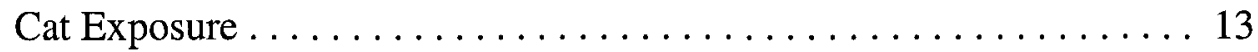

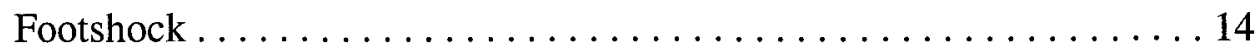

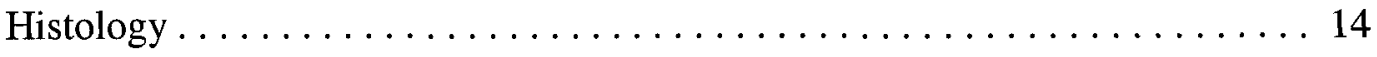

Behavioral Analysis . ............................... 15

Chapter 3: Results: Dorsal Hippocampus $\ldots \ldots \ldots \ldots \ldots \ldots \ldots \ldots \ldots \ldots \ldots$

Histology $\ldots \ldots \ldots \ldots \ldots \ldots \ldots \ldots \ldots \ldots \ldots \ldots \ldots \ldots, 16$

Unconditioned Behavior Results ....................... 16

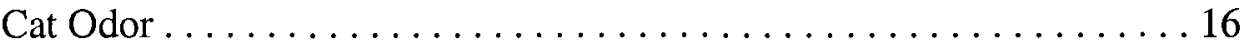


Cat Exposure ............................. 17

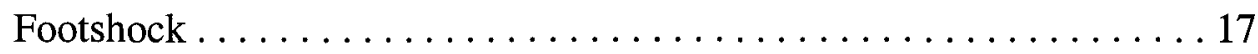

Conditioned Behavior Results $\ldots \ldots \ldots \ldots \ldots \ldots \ldots \ldots \ldots \ldots \ldots$

Cat Odor Cue + Context Conditioning $\ldots \ldots \ldots \ldots \ldots \ldots \ldots \ldots \ldots$

Cat Exposure Context conditioning $\ldots \ldots \ldots \ldots \ldots \ldots \ldots \ldots \ldots$

Footshock Context Conditioning ..................... 19

Chapter 4: Discussion: Dorsal Hippocampus . ................. 20

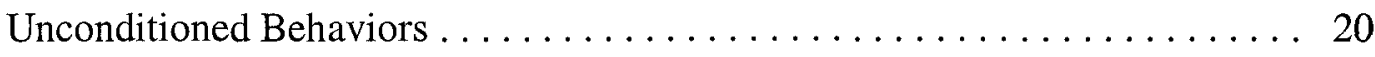

Conditioned Behaviors $\ldots \ldots \ldots \ldots \ldots \ldots \ldots \ldots \ldots \ldots \ldots \ldots \ldots \ldots \ldots \ldots \ldots, 22$

Chapter 5: Methods and Procedures: Ventral Hippocampus ............... 23

Animals $\ldots \ldots \ldots \ldots \ldots \ldots \ldots \ldots \ldots \ldots \ldots \ldots \ldots \ldots \ldots, 23$

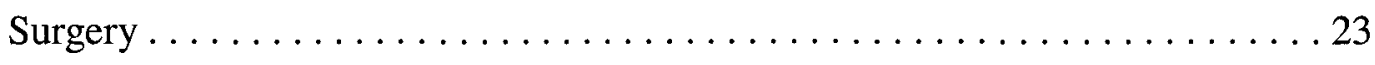

Behavioral Testing Procedures $\ldots \ldots \ldots \ldots \ldots \ldots \ldots \ldots \ldots \ldots \ldots, 24$

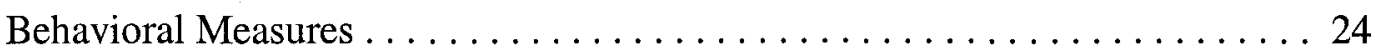

Behavioral Tests ................................ 25

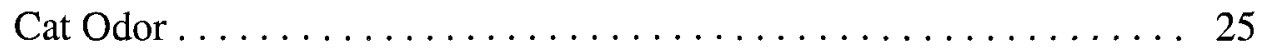

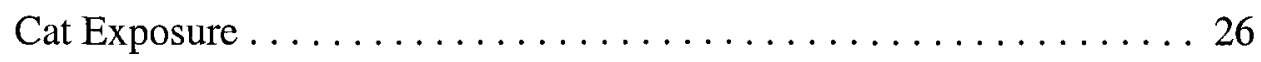

Footshock ................................. 27

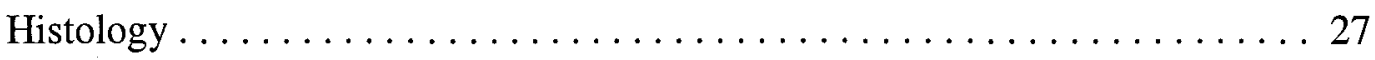

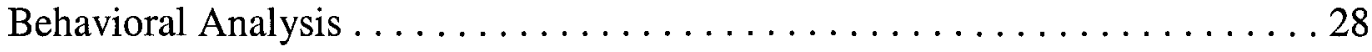

Chapter 6: Results: Ventral Hippocampus . . . . . . . . . . . . . . . . . . . . . 29

Unconditioned Behavior Results ....................... 29

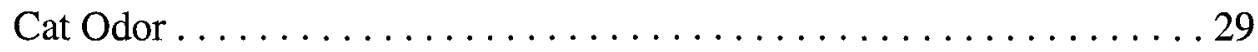


Cat Exposure ............................. 30

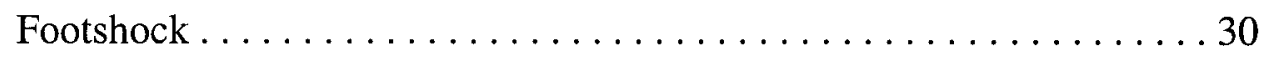

Conditioned Behavior Results . . . . . . . . . . . . . . . . . . 31

Cat Odor Cue + Context Conditioning $\ldots \ldots \ldots \ldots \ldots \ldots \ldots \ldots \ldots \ldots \ldots$

Cat Exposure Context Conditioning . . . . . . . . . . . . . 32

Footshock Context Conditioning $\ldots \ldots \ldots \ldots \ldots \ldots \ldots \ldots \ldots \ldots \ldots$

Chapter 7: Discussion: Ventral Hippocampus $\ldots \ldots \ldots \ldots \ldots \ldots \ldots \ldots \ldots \ldots \ldots \ldots$

Unconditioned Behaviors ............................. 33

Conditioned Behaviors ................................. 39

Chapter 8: Discussion: General ......................... 42

Table 1: DH Cat Odor Results ........................... 44

Table 2: DH Cat Exposure Results ....................... 44

Table 3: DH Footshock Results $\ldots \ldots \ldots \ldots \ldots \ldots \ldots \ldots \ldots \ldots \ldots \ldots \ldots$

Table 4: VH Cat Odor Results $\ldots \ldots \ldots \ldots \ldots \ldots \ldots \ldots \ldots \ldots \ldots \ldots \ldots$

Table 5: VH Cat Exposure Results ........................ 46

Table 6: VH Footshock Results ............................. 46

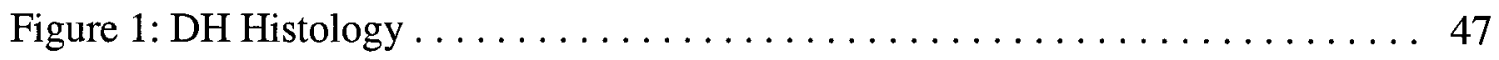

Figure 2: VH Histology $\ldots \ldots \ldots \ldots \ldots \ldots \ldots \ldots \ldots \ldots \ldots \ldots \ldots \ldots$

Figure 3: VH Cat Odor Results . . . . . . . . . . . . . . . . . . . 49

Figure 4: VH Cat Exposure Results ......................... 50

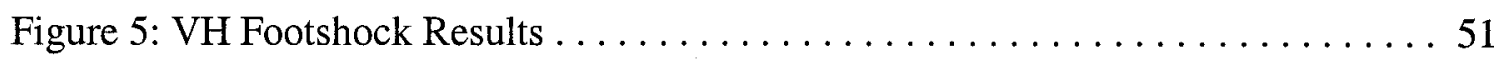

References........................................ 52 


\section{LIST OF TABLES}

$\underline{\text { Table }}$

$\underline{\text { Page }}$

1. DH Cat Odor Results ....................... 44

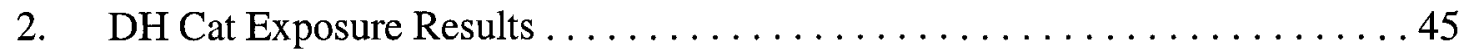

3. DH Footshock Results ........................ 46

4. VH Cat Odor Results ...................... 47

5. VH Cat Exposure Results $\ldots \ldots \ldots \ldots \ldots \ldots \ldots \ldots \ldots \ldots \ldots$

6. VH Footshock Results $\ldots \ldots \ldots \ldots \ldots \ldots \ldots \ldots \ldots \ldots \ldots \ldots \ldots$ 


\section{LIST OF FIGURES}

Figure $\quad \underline{\text { Page }}$

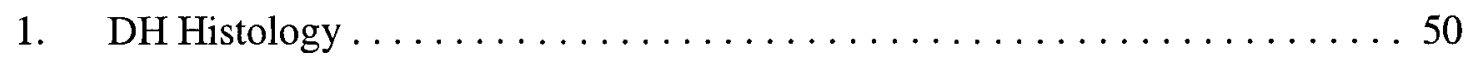

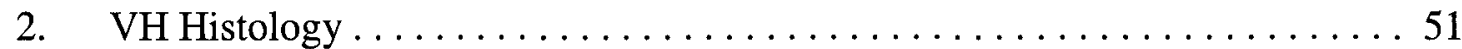

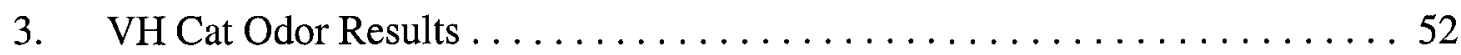

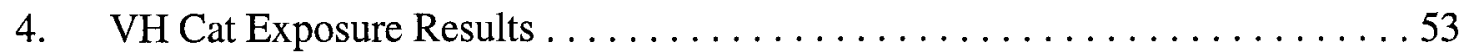

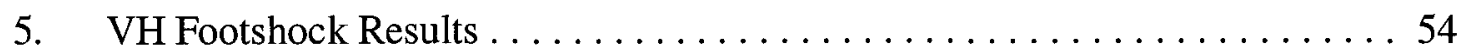




\section{CHAPTER 1. INTRODUCTION}

\subsection{Unconditioned Defensive Behavior}

It is widely believed that the hippocampus is involved in certain forms of learning and memory (Manns, Hopkins \& Squire, 2003; Burgess, Maguire \& O'Keefe, 2002; Jarrard, 1993; Squire, 1992; O’Keefe \& Nadel, 1978), particularly explicit or declarative memory (Squire, Stark \& Clark, 2004). The hippocampus participates in spatial navigation and relational learning (O'Keefe and Nadel, 1978), functioning to process and encode contextual information related to spatial location (Jarrard, 1993). Episodic memory consisting of memories for episodes or past perceptions of events, organized in a particular time and space, also depends on hippocampal functioning (Mann et al., 2003; Burgess et al., 2002). Finally, the hippocampus is involved in the formation of semantic memory or memory for facts and knowledge (Burgess et al., 2002; Squire, 1992).

In addition to a role in learning and memory, it has become increasingly apparent that the hippocampus is involved in the regulation of behavior (Bannerman, Rawlins, McHugh, Deacon, Yee, Bast, Zhang, Pothuizen and Feldon, 2004). Previous research has implicated hippocampal functioning in the modulation of anxiety (Degroot and Treit, 2004,2003 ) and the expression of innate defensive responses to various threat stimuli (Canteras 2002; Blanchard, Blanchard, Lee, \& Fukunaga, 1977; Blanchard \& Blanchard, 1972a; Kim, Kim, Kim, Kim, Chang, Kim, and Lee, 1971; Blanchard, Blanchard, \& Fial, 1970). The Blanchards (1972a) showed that complete electrolytic hippocampal lesions reduced specific unconditioned defensive responses (freezing) to, and increased active avoidance of, a natural predatory threat stimulus (cat), despite having no effect on general activity. Furthermore, complete electrolytic hippocampal lesions increased active 
avoidance of, and decreased defensive fear/immobility (crouching) to, an electrified shock probe (Blanchard et al., 1970), while reducing the duration of freezing during the administration of continuous foot-shocks (Blanchard et al., 1977). Kim et al. (1971) demonstrated that complete hippocampal aspirations also reduced fear expression in rats, indicated by reduced freezing and increased food consumption, during exposure to a live cat. Collectively, this early research indicates that the hippocampus may be involved in modulating the expression of unconditioned defensive behaviors during exposure to both predatory and painful threat stimuli.

Recent research by Degroot and Treit (2002) provide additional evidence for hippocampal involvement in regulating the expression of unconditioned defensive responses. Specifically, hippocampal cholinergic and septal GABAergic systems appear to interact to modulate anxious behavior in the elevated plus-maze (EPM) and shock probe-burying test (SPBT). Increasing the levels of acetylcholine in either the dorsal or ventral hippocampus by injecting the acetylcholinesterase inhibitor physostigmine decreased anxiety-like behavior in the EPM, measured by increased open arm exploration (increased number of entries and duration of time spent in the open arms), and decreased burying behavior in the shock probe-burying test (Degroot and Treit, 2003; Degroot and Treit, 2002; Degroot, Kashluba and Treit, 2001). Finally, complete, dorsal and ventral hippocampal electrolytic lesions decreased anxiety-like responses assessed in the EPM and social interaction test (Bannerman, Deacon, Offen, Friswell, Grubb and Rawlins, 2002), while complete excitotoxic hippocampal lesions decreased anxiolytic-like responses in the three tests of anxiety, zero-maze, social interaction and hyponeophagia (Deacon, Bannerman and Rawlins, 2002). 
Additionally, the dorsal and ventral poles of the hippocampus seem to play key roles in modulating passive avoidance of painful threat stimuli. Injections of physostigmine into the VH increased the number of shocks incurred in the SPBT (Degroot and Treit, 2002; Treit and Menard, 1997), while reversible lesions of the DH using tetrodotoxin, a sodium channel blocker, produced a similar effect (Degroot and Treit, 2004).

Collectively these results indicate that the hippocampus may help regulate the expression of various unconditioned behaviors in certain animal tests of defense.

Recent work by Canteras (2002) regarding the medial hypothalamic defensive system (MHZ defensive system) suggests a mechanism by which the hippocampus could function as part of a neural circuit subserving the expression of unconditioned defensive behaviors. Exposure to cat odor (Dielenberg, Hunt and McGregor, 2001; Dielenberg and McGregor, 2001) or to a live cat (Canteras 2002; Canteras, Chiavegatto, Ribeiro do Valle and Swanson, 1997) increases fos immunoreactivity, a sensitive cellular marker for neural activation, in the MHZ defensive system consisting of the dorsal premammillary nucleus ( $\mathrm{PMd})$, anterior hypothalamus ( $\mathrm{AHN}$ ) and the dorsal medial portion of the ventral medial hypothalamus (VMHdm) (Canteras, 2002; Risold, Canteras and Swanson, 1994; Canteras and Swanson, 1992b). Lesions to selective components of the MHZ defensive system disrupt the responsivity of rats to various predatory threat stimuli. Lesions of the PMd dramatically reduced freezing to a live cat (Markham, Blanchard, Canteras, Cuyno and Blanchard, 2004; Blanchard, Li, Hubbard, Markham, Yang, Takahashi and Blanchard, 2003; Canteras et al., 1997) as well as freezing and avoidance of predatory odors (Markham et al., 2004; Blanchard et al., 2003). 
The three highly interconnected structures of the MHZ defensive system (AHN, VMHdm \& PMd) are connected to various forebrain, midbrain and hindbrain structures, including the amygdala, bed nucleus of the stria terminalis (BNST), prefrontal cortex (PFC), periaqueductal gray (PAG), septum and hippocampus, structures all believed to be involved in threat-linked behavior (Canteras 2002; Canteras, Ribeiro-Barbosa \& Comoli, 2001). Tract-tracing studies have shown that the hippocampus sends projections to the MHZ defensive system via two main pathways. First, the hippocampus sends projections from intermediate regions of $\mathrm{CA} 1$ and the subiculum through the dorsal region of the ventrolateral zone of the rostral part of the lateral septal nucleus (LSrvld) (Risold and Swanson, 1997). The LSrvld then projects to the MHZ defensive system through the AHN \& PMd (Canteras et al., 2001, Comoli, Ribeiro-Barbosa and Canteras, 2001; Risold and Swanson, 1997). This pathway is potentially involved in regulating defensive behavioral reactions as the LSrvld contains GABAergic neurons that provide inhibitory inputs to circuits mediating defensive reactions (AHN \& PMd). Furthermore, lesions to the LSrvld produce hyperdefensiveness or "septal rage" (Albert and Chew, 1980), presumably stemming from the loss of inhibition from the LSrvld. Secondly, the hippocampus sends projections from intermediate regions of CA1 and the subiculum through the lateral, posterior basomedial and posterior basolateral amygdalar nuclei (Pikkarainen, Ronkko, Savander, Insausti and Pitkanen, 1999; Petrovich, Risold and Swanson, 1996), which project to the BNST. The BNST then projects to the MHZ defensive system through the AHN. These amygdalar-hippocampal connections are potentially involved in modulating unconditioned defensive behaviors, serving to integrate olfactory, insular, prefrontal and temporal cortical processing to influence the 
MHZ defensive system (Petrovich, Canteras and Swanson, 2001; Pikkarainen et al, 1999; Petrovich et al., 1996). Taken as a whole, these neural connections suggest that the MHZ defensive system could be mediated by a septo-hippocampal domain (Canteras, 2002).

\subsubsection{Dorsal and Ventral Hippocampal Specificity}

The majority of the research investigating the role of the hippocampus in the elicitation of behavior has focused on the involvement of the whole structure. However, there is considerable evidence to suggest that the hippocampus should be functionally differentiated into dorsal and ventral poles (Bannerman et al., 2004; Risold and Swanson, 1996). While research implicating the $\mathrm{DH}$ as a structure involved in the selection of unconditioned defensive behaviors is mainly limited to its effects on passive avoidance (Degroot and Treit, 2002; 2004), there is some evidence suggesting that the DH may play a specific role in regulating active responses in EPM. In addition to increasing levels of acetylcholine (Degroot and Treit, 2002; Degroot et al., 2001), micro-infusions of midazolam into the $\mathrm{DH}$ significantly increased both the duration of time spent in the open arms and the number of open-arm entries, without a concurrent increase in general activity (Menard and Treit, 2001).

While there is relative paucity of research demonstrating the specific involvement of the $\mathrm{DH}$ in modulating unconditioned defensive behaviors, there is a substantial amount implicating the VH. Degroot and Treit (2004) demonstrated an anxiolytic-like effect of increased open arm exploration in the EPM and decreased burying behavior in the SPBT following reversible VH lesions using tetrodotoxin, an effect not seen with DH lesions. This finding was interpreted as an anxiolytic-like effect as no differences in general 
activity were detected between groups, measured by the total number of open arm entries. Similarly, rats with selective excitotoxic ventral, but not dorsal hippocampal lesions displayed reduced corticosterone secretion following confinement to a brightly lit chamber, and entered and spent more time in the open arms of the EPM, effects not seen in DH or amygdala lesioned animals (Kjelstrup, Tuvnes, Steffenach, Murison, Moser and Moser, 2002). This outcome was also not attributable to general increases in activity as both groups entered the arms that were closed during initial testing as frequently during a subsequent trial conducted with all four arms closed. These results were also not the result of spatial navigation deficits as $\mathrm{VH}$ lesioned animals showed no deficits in the Morris water maze, an expected effect as sensory association cortices primarily project to the DH (Pikkarainen et al., 1999). Trivedi and Coover (2004) found that rats with electrolytic lesions specific to the ventral, but not dorsal, hippocampus failed to avoid the open arms of an elevated t-maze, while excitotoxic VH lesions reduced anxiety-like behaviors in the social interaction, light/dark, hyponeophagia and successive alley tests (McHugh, Deacon, Rawlins and Bannerman, 2004). Finally, VH NMDA lesioned animals displayed a reduction in freezing following the delivery of an unconditioned, unsignalled footshock, and entered the black box sooner during a version of the light/dark exploration test, despite no detectable differences in general locomotor activity in an open field (Bannerman, Grubb, Deacon, Yee, Feldon and Rawlins, 2003). Collectively, these studies indicate that the brain system (or systems) associated with fear and/or anxiety likely includes the ventral and not dorsal hippocampus. 


\subsection{Conditioned Defensive Behavior}

As well as a possible role in modulating the elicitation of defensive responses to unconditioned threat stimuli, the hippocampus also seems to be involved in contextual conditioning. Several functional theories have proposed that the hippocampus is involved in the acquisition, consolidation and retrieval of a conjunctive representation of a context, which is based on multimodal stimuli including, sensory, temporal and spatial information (Matus-Amat, Higgins, Barrientos and Rudy, 2004; Sanders, Wiltgen and Fanselow, 2003; Anagnostaras, Gale and Fanselow, 2001; O'Keefe and Nadel, 1978). Researchers have further suggested a specific time limited role for the $\mathrm{DH}$ in the acquisition, consolidation and retrieval of contextual fear (Matus-Amat et al., 2004; Sanders et al., 2003; Barrientos, O'Reilly and Rudy, 2002; Fanselow, 2000; Maren, Aharonov and Fanselow, 1997). Infusions of scopolamine into the DH immediately prior to conditioning produces deficits in contextual fear (Gale, Anagnostaras and Fanselow, 2001), while NMDA lesions made one week prior to training produce no deficits (Richmond, Yee, Pouzet, Veenman, Rawlins, Feldon and Bannerman, 1999; Maren et al., 1997). Thus, while pretraining electrolytic lesions produce modest reductions in fear conditioning, this effect likely results from damage to fibers of passage. Conversely, permanent hippocampal lesions made 1-28 days post-training, but not after (Maren et al., 1997), attenuate context conditioning, while reversible inactivation of the $\mathrm{DH}$ using tetrodotoxin up to, but not after ninety-minutes following conditioning disrupt consolidation of contextually conditioned fear, evidenced by a reduction in freezing (Sacchetti, Lorenzini, Baldi, Tassoni and Bucherelli, 1999). Thus after consolidation, the 
memory for the context is stored outside the hippocampus, presumably the neocortex (Matus-Amat, 2004; Fanselow, 2000).

Despite considerable evidence for DH involvement, research also indicates that the VH may participate in the formation of contextual fear. Trivedi and Coover (2004) demonstrated that post-training electrolytic lesions of the ventral, but not dorsal hippocampus, significantly reduced the expression of freezing to both context and tone conditioned stimuli. Similarly, ventral hippocampal reversible lesions using tetrodotoxin and infusions of the both the GABA receptor antagonist muscimol (Bast, Zhang and Feldon, 2001) and an NMDA receptor antagonist MK-801, all reduced the expression of conditioned fear (Zhang, Bast and Feldon, 2001). Finally, pretraining excitotoxic ventral, but not dorsal, hippocampal lesions produced deficits in conditioned freezing during a signaled shock procedure (Richmond et al., 1999). Collectively, these findings suggest that in addition to the $\mathrm{DH}$, the $\mathrm{VH}$ may constitute a neural structure subserving contextual fear conditioning.

Collectively, these studies clearly implicate hippocampal involvement in contextual fear conditioning; however, they typically utilized traditional Pavlovian fear conditioning techniques. These include placing an animal in a chamber and delivering either paired or unpaired repeated footshocks. In this situation, rats will quickly condition to the painful stimulus and display freezing in the absence of footshocks. Therefore, given these testing parameters, the majority of research implicating the hippocampus in contextual fear conditioning has only assessed freezing in response to painful threat stimuli. In addition to elicitation through pain, conditioned defensive behaviors including risk assessment, avoidance and freezing can be induced by exposing rats to a predatory threat stimulus, 
such as cat odor or a live cat (Blanchard, Blanchard \& Hori, 1989). After exposure to the unconditioned predatory stimulus, rats tested subsequently in the same context without the threat stimulus, will then exhibit conditioned defensive behaviors (Blanchard, Yang, $\mathrm{Li}$, Gervacio and Blanchard, 2001). These behavioral responses allow for the careful scrutiny of the neurological substrates controlling both innate (unconditioned) and learned (conditioned) defensive behaviors to both predatory and painful threat stimuli.

Therefore, the current study sought to investigate a possible dual role of the hippocampus in modulating the expression of both unconditioned and conditioned defensive behaviors by examining the effects of bilateral dorsal and ventral hippocampal lesions during exposure to both predatory (cat odor and cat exposure) and painful (footshock) threat stimuli. It was hypothesized that lesions of the DH would significantly reduce conditioned defensive behaviors, including risk assessment, avoidance and freezing, while VH lesions would significantly reduce these same unconditioned behaviors during all three tests of defense. 


\section{CHAPTER 2. METHODS AND PROCEDURES: DORSAL HIPPOCAMPUS}

\subsection{Animals}

Subjects were 29 male Long-Evans hooded rats born and reared from the Snyder Hall breeding colony at the University of Hawaii. Animals weighed between 350 and 520 grams at the time of surgery. Following weaning (21 days), all animals were singly housed under controlled temperature $(23 \mathrm{C})$ and illumination $(12: 12$ hour light/dark cycle, with lights on at $0600 \mathrm{~h}$ ) with free access to food and water.

\subsection{Surgery}

Prior to surgery, animals were randomly assigned to either a DH experimental-lesion group $(\mathrm{N}=14)$, or to a sham operated-control group $(\mathrm{N}=15)$. Subjects were deeply anesthetized with an intraperitoneal injection of sodium pentobarbital $(65 \mathrm{mg} / \mathrm{kg} \mathrm{IP})$ and were mounted in a stereotaxic apparatus (David Kopf Instruments, Tujunga, CA). The scalp was incised and retracted, and the head was positioned to place Bregma and Lambda in the same horizontal plane. Two sets of small burr holes ( $2.0 \mathrm{~mm}$ in diameter) were drilled in the skull bilaterally in order to place a stainless steel cannula (28G, Plastics One) into the DH. The cannula was connected to a 10 ul micro syringe (Hamilton) mounted in an infusion pump (Harvard Apparatus) to control the rate of infusion $(0.04 \mathrm{ul} / \mathrm{min})$. DH-lesioned animals received two separate bilateral infusions $(0.30 \mathrm{ul})$ of a $.1 \%$ ibotenic acid solution (Sigma) in the anterior and posterior regions of the DH (Paxinos and Watson, 1998): anterior $(-2.8 \mathrm{~mm}$ posterior to bregma, $+/-1.6 \mathrm{~mm}$ from the midline and $-3.3 \mathrm{~mm}$ ventral from the surface of the brain); posterior $(-4.2 \mathrm{~mm}$ posterior to bregma, $+/-2.6 \mathrm{~mm}$ from the midline and -3.0 ventral from the surface of the 
brain). Following the infusion, the cannula remained in place for an additional five minutes to ensure complete diffusion of the ibotenic acid. For sham-operated controls, the cannula was placed $0.5 \mathrm{~mm}$ above the DH and no ibotenic acid was infused. Thus, any differences found between lesion and control animals could not be attributed to cortical damage in the experimental group. Following surgery, animals were returned to their home cage and allowed a one-week recovery time prior to the start of behavioral testing.

\subsection{Behavioral Testing Procedures}

Behavioral testing was conducted between 0900-1300 hours in the following sequence: Cat Odor Test - Cat Exposure Test - footshock, each beginning on consecutive days. Each of the three behavioral tests measured both unconditioned and conditioned defensive behaviors. Unconditioned defensive behaviors were assessed during (cat odor and cat exposure), or immediately following the exposure to the threat stimulus (footshock). Conditioned defensive behaviors were assessed 24 hours after exposure to the threat stimulus in the same context. Between every subject trial, each apparatus was thoroughly cleaned using a 5\% ethanol solution.

\subsection{Behavioral Measures}

All trials were recorded on videotape for subsequent behavioral analysis from a highly trained observer blind to experimental conditions. The specific dependent measures in each condition varied, but are listed following the description of each behavioral test. The specific behaviors measured included: 1) freezing - complete cessation of movement 
other than respiration; 2) stretch approach - forward ambulation with flat back and stretched neck; 3) stretch attend - standing on all four paws with flat back and stretched neck; 4) rearing - standing on rear paws with forepaws raised off the ground; 5) standing - both fore and hind limbs extended ; 6) crouching - elevation of the forelimbs off the floor and arching of the back; 7) grooming - movement of forepaws or tongue over the body; 8) sniffing - olfactory investigation evidenced by vertical or lateral head movements; scoring initiated when nose visibly moved more than $1 \mathrm{~cm}$; 9) transits - line crossing between far, medium and near locations; measured as any movement from one marked section of the apparatus to another; 10) Contact - direct contact with the wire mesh or block stimulus; measured as direct paw or head contact; 11) Location - duration of time spent in the near, medium or far compartment relative to the threat stimulus (cat odor: block; cat exposure: wire-mesh divider).

\subsection{Behavioral Tests}

\subsubsection{Cat Odor}

The test apparatus consisted of a white Plexiglas runway $(100 \times 12 \times 50 \mathrm{~cm})$ with a clear Plexiglas front panel to permit observation and videotaping. A cloth-wrapped solid plastic block $(9 \times 9 \times 2 \mathrm{~cm})$ was rubbed for five minutes against the fur of laboratoryhoused domestic male cat for three consecutive days and was then stored in a Ziploc plastic bag until serving as the cat odor stimulus. Both control and experimental animals were habituated to the apparatus for three consecutive days without the presence of an odor block. On the fourth day (unconditioned behavior test), the cat odor block was placed at one end of the runway and a subject was placed at the opposite end, facing 
away from the cat odor block. Twenty-four hours later each subject was retested in the same apparatus without the cat odor stimulus (conditioned behavior test). A clothwrapped solid plastic block never exposed to cat odor served as the cue during the conditioned behavioral test. All sessions were ten minutes in duration and were conducted under red light in order to ensure that the odor stimulus remained as ambiguous as possible.

The test apparatus was divided into three equal sections, far, medium and near the odor stimulus. Measures regarding each subject's location were investigated and included transits between sections and contacts with the odor stimulus (block). Behavioral measures were also examined and included freezing, a composite of the two risk assessment measures (stretch attend and stretch approach), and grooming, head movement, rearing, standing and crouching.

\subsubsection{Cat Exposure}

The test apparatus consisted of two adjacent subject chambers $(50 \times 20 \times 30 \mathrm{~cm})$ separated by an opaque white Plexiglas wall, with a wire-mesh screen separating these chambers and the adjoining cat compartment $(55 \times 40 \times 35 \mathrm{~cm})$. Two subjects were simultaneously placed, one in each subject chamber, facing away from the cat compartment (the same cat used in the cat odor test). Following a five-minute pre-cat exposure period, the cat was placed in the cat compartment for ten-minutes (unconditioned behavior test). Twenty-four hours later, subjects were retested in the same apparatus without the cat stimulus (conditioned behavior test). Each test was conducted under white light to ensure the cat stimulus was as unambiguous as possible. 
The test apparatus was divided into three equal sections, far, medium and near the cat stimulus. Measures regarding each subject's location were investigated and included transits between sections and contacts with the wire-mesh divider. Behavioral measures were also examined and included freezing, a composite of the two risk assessment measures (stretch attend and stretch approach), and grooming, head movement, rearing, standing and crouching.

\subsubsection{Footshock}

The footshock test apparatus $(25 \times 25 \times 50 \mathrm{~cm})$ was constructed of black Plexiglas with a clear front panel to permit observation and videotaping. A scrambled electric foot shock from a LaFayette-82401 generator was delivered via a stainless steel grid floor to serve as the painful threat stimulus. After a three-minute habituation period, three footshocks (1.0 mA, $1 \mathrm{~s}$ duration) were delivered at one-minute intervals, followed by a five-minute observation period (unconditioned behavior test). Twenty-four hours later each subject was retested in the same apparatus without the shock stimulus (conditioned behavior test). Each test was conducted under white light in order to ensure that the shock stimulus remained as unambiguous as possible. Behavioral measures included freezing, grooming, head movement, rearing, standing and crouching.

\subsection{Histology}

Following the completion of behavioral testing, histological verification of lesion placement was performed. All Subjects were overdosed with sodium pentobarbital and were perfused transcardially with $0.9 \%$ saline followed by $10 \%$ formalin. Following 
extraction from the skull, brains were placed into a $10 \%$ formalin solution for 48 hours and then transferred to a $30 \%$ sucrose-formalin solution until blocking (at least 48 hours) and sectioning on a cryostat (Leica). A series of coronal sections were collected ( $50 \mathrm{um}$ thick taken every $100 \mathrm{um}$ ) and after drying ( 24 hours), the sections were thionin-stained in order to visualize cell damage to the stained brain sections using a microscope (Leica).

To assess the amount of damage to the $\mathrm{DH}$, sections were mapped on schematic representations employing the rat brain atlas of Swanson (1998).

\subsection{Behavioral Analysis}

Independent Student's t-tests were performed on each dependent measure (unconditioned and conditioned), for both the frequency and duration of each measure. Alpha was set at 0.05 . Mann-Whitney $U$ tests were used to assess the statistical reliability of skewed data. 


\section{CHAPTER 3. RESULTS: DORSAL HIPPOCAMPUS}

\subsection{Histology}

Figure 1 presents serial histological reconstructions of excitotoxic DH lesions for rats included in the statistical analyses for each experimental condition. Four rats were excluded from statistical analysis due to partial or complete unilateral sparing of cells in the DH. All of the rats included the DH experimental group suffered complete, bilateral lesions of the $\mathrm{DH}$ formation, with minimal damage to surrounding structures. This minimal damage was largely restricted to cannula tract marks and was therefore equivalent to the damage found in the sham-lesioned controls. Nearly all of the remaining cells were found in the most posterior-lateral portions of the DH (CA-2 region). Thus, most of the $\mathrm{DH}$ was destroyed including virtually all of the granular neurons in the dentate gyrus, as well all of the pyramidal cells in regions CA-1 and CA-3. These results are comparable in size to those obtained by others using similar excitotoxic methods (Maren et al., 1997).

\subsection{Unconditioned Behavior Results}

\subsubsection{Cat Odor}

The frequencies and durations of each location and behavioral measure during the cat odor test for both the DH-lesioned and sham-operated control groups are presented in Table 1. With respect to measures of location, lesion and sham animals did not significantly differ in the duration of contact with the odor block, $t(23)=1.43, p>0.05$, or the duration of time spent near, medium or far from the stimulus, $t(23)=0.81, p>$ $0.05, t(23)=1.13, p>0.05, t(23)=0.31, p>0.05$, respectively. The durations of time 
spent engaged in freezing, $t(23)=0.61, p>0.05$, sniffing $t(23)=0.26, p>0.05$, or risk assessment, $t(23)=0.95, p>0.05$, were also not significantly different between groups. Neither group differed on two measures of posture, standing, $t(23)=0.74, p>0.05$, and crouching, $t(23)=0.62, p>0.05$, or spent more time grooming, $t(23)=1.11, p>0.05$, or rearing, $t(23)=0.89, p>0.05$. There was no difference in the number of transits between the two groups, $t(23)=0.08, p>0.05$.

\subsubsection{Cat Exposure}

Table 2 presents the frequencies and durations of each behavioral and location measure during the cat exposure test for both the DH-lesioned and sham-operated control groups. With respect to location measures, nether group significantly differed in the duration of contact with the wire mesh, $t(23)=0.81, p>0.05$, or the duration of time spent near, medium or far from the predatory threat stimulus, $t(23)=0.41, p>0.05, t(23)$ $=1.19, p>0.05, t(23)=1.41, p>0.05$, respectively. The durations of time spent engaged in freezing, $t(23)=0.15, p>0.05$, and sniffing $t(23)=0.22, p>0.05$, were also not significantly different, and neither group engaged in risk assessment. Durations of time spent standing, $t(23)=0.33, p>0.05$, crouching, $t(23)=0.02, p>0.05$, grooming, $t(23)=0.81, p>0.05$, or rearing, $t(23)=0.83, p>0.05$, were not significantly different, and there was no difference in the number of transits, $t(23)=1.47, p>0.05$.

\subsubsection{Footshock}

The durations of each behavior during the post footshock test for both the DHlesioned and sham-operated control groups are presented in table 3. Subjects in both 
groups did not significantly differ in levels of freezing, $t(23)=0.79, p>0.05$, or sniffing, $t(23)=0.54, p>0.05$. Additionally, there were no significant differences between

groups in the amount of grooming, $t(23)=1.24, p>0.05$, standing, $t(23)=0.34, p>0.05$, crouching, $t(23)=0.96, p>0.05$, or rearing, $t(23)=0.03, p>0.05$.

\subsection{Conditioned Behavior Results}

\subsubsection{Cat Odor Cue + Context Conditioning}

The frequencies and durations of each location and behavioral measure during the cat odor cue + conditioning test for both the $\mathrm{DH}-$ lesioned and sham-operated control groups are presented in table 1. For measures of location, lesion and sham operated controls did not significantly differ in duration of contact with the non-odor block, $t(23)=0.61, p>$ 0.05 , or the duration of time spent near, medium or far from the stimulus, $t(23)=0.61, p$ $>0.05, t(23)=1.02, p>0.05, t(23)=0.80, p>0.05$, respectively. Durations of freezing, $t(23)=1.11, p>0.05$, sniffing $t(23)=1.93, p>0.05$, and risk assessment, $t(23)=1.44, p$ $>0.05$, were also not significantly different between groups. Neither group displayed significant differences on durations of standing, $t(23)=0.78, p>0.55$, crouching, $t(23)=$ $0.10, p>0.05$, grooming, $t(23)=0.19, p>0.05$, or rearing, $t(23)=0.67, p>0.05$.

Finally, there was no difference between the two groups in the number of transits, $t(23)=$ $0.69, p>0.05$

\subsubsection{Cat Exposure Context Conditioning}

Table 2 presents the frequencies and durations of each behavioral and location measure during the cat exposure context conditioning test for both the experimental and 
control groups. Nether lesion or sham animals significantly differed in the duration of contact with, $t(23)=1.57, p>0.05$, or the time spent near, medium or far from the wire mesh, $t(23)=0.19, p>0.05, t(23)=1.16, p>0.05, t(23)=0.04, p>0.05$, respectively. Groups did not significantly differ in durations of freezing, $t(23)=0.74, p>0.05$, sniffing $t(23)=0.73, p>0.05$, or risk assessment, $t(23)=0.92, p>0.05$. Group differences for standing, $t(23)=0.56, p>0.05$, crouching, $t(23)=0.10, p>0.05$, grooming, $t(23)=0.10, p>0.05$, and rearing, $t(23)=0.76, p>0.05$, were also not significantly different, and there was no difference in the number of transits, $t(23)=1.12$, $p>0.05$.

\subsubsection{Footshock Context Conditioning}

Durations of each behavioral measure during the footshock context conditioning test for both the DH-lesioned and sham-operated control groups are presented in Table 3. No significant group differences were detected in levels of freezing, $t(23)=0.80, p>0.05$, or sniffing, $t(23)=1.01, p>0.05$. Additionally, there were no significant differences between groups in levels of grooming, $t(23)=1.81, p>0.05$, standing, $t(23)=0.10, p>$ 0.05 , crouching, $t(23)=0.61, p>0.05$, or rearing, $t(23)=1.31, p>0.05$. 


\section{CHAPTER 4. DISCUSSION: DORSAL HIPPOCAMPUS}

\subsection{Unconditioned Behaviors}

Results of the present study help clarify the role of the DH in modulating unconditioned defensive behaviors to both painful and predatory threat stimuli. In all three testing paradigms, DH-lesion and sham-operated controls did not significantly differ on any behavioral measure, including durations of freezing or risk assessment, nor did subjects differ in levels of avoidance during the cat odor or cat exposure tests. Therefore, results seem to indicate that the $\mathrm{DH}$ is not involved in modulating defensive responses to unconditioned predatory or painful threat stimuli.

These results suggest that the work by the Blanchards and colleagues $(1970 ; 1972 \mathrm{a}$; 1977), Kim et al. (1971) and Deacon et al. (2002), which implicated hippocampal involvement in the expression of unconditioned defensive responses, could be specific to the ventral pole of the hippocampus. Another possible explanation for the earlier results is that these studies utilized either electrolytic lesions or aspirations. These techniques produce damage to fibers of passage, damaging glutamatergic neurons projecting from the hippocampus to the nucleus accumbens (Totterdell and Smith, 1989), which could potentially increase ambulation, an effect that could explain the mutual finding of decreased levels of freezing. The current study employed excitotoxic methods that spare fibers of passage, which could account for the null effect on levels of freezing and provide evidence that the $\mathrm{DH}$ has no effect on the elicitation of defensive behaviors during these three unconditioned tests.

The null findings regarding the role of the DH in regulating the responsivity of rats to painful and predatory threat stimuli are consistent with studies that implicate the ventral, 
not dorsal hippocampus in modulating anxiolytic like-responses in the EPM and SPBT (Degroot and Treit, 2003). Reversible lesions specific to the ventral pole of the hippocampus increased open arm exploration and decreased burying behavior, while dorsal lesions failed to produce similar effects. Furthermore, electrolytic lesions specific to the $\mathrm{DH}$ failed to increase open arm exploration or decrease burying behavior in the EPM and SPBT, respectively (Treit and Menard, 1997). While Bannerman et al. (2002) found anxiolytic effects in the social interaction and plus-maze tests with both VH and DH electrolytic lesions, they reported hyperactivity in only the DH group. When excitotoxic lesions were utilized in the same testing procedures, anxiolytic effects were limited to only the VH (McHugh, Deacon, Rawlins, and Bannerman, 2004). Lastly, DH infusions of scopolamine failed to alter unconditioned responses following footshock (Gale et al., 2001). Therefore, the anxiolytic-like effects reported using electrolytic lesions to the DH could have resulted from damage to fibers of passage.

Results from the current study suggesting a possible role for the ventral, not dorsal hippocampus in the elicitation of defensiveness, is consistent with findings from tracktracing studies, which indicate that the CA1 regions of the $\mathrm{VH}$ and the ventral subiculum send more projections to the LSrvld (Petrovich et al., 2001; Risold \& Swanson, 1996). Furthermore, the ventral poles of the CA1 regions, subiculum and entorhinal cortex have stronger bilateral projections with the basomedial amygdala than the dorsal pole of the hippocampus (Petrovich et al., 2001; Canteras \& Swanson, 1992a). Thus, the ventral, not dorsal hippocampus likely plays a stronger role in the selection of innate defensive responses through its connections with the amygdala and MHZ defensive system. 


\subsection{Conditioned Behaviors}

Results from the current study also help clarify the role of the $\mathrm{DH}$ in contextual fear conditioning to both predatory and painful threat stimuli. In the cat odor, cat exposure and shock tests for conditioned defensive behaviors, DH-lesion and sham-operated controls did not significantly differ in levels of freezing, risk assessment or any other behavioral measure. Therefore, results seem to indicate that the $\mathrm{DH}$ is not involved in the neural circuit or circuits that elicit conditioned defensive responses to predatory or painful threat stimuli.

Upon careful review of the literature, the null findings regarding the role of the $\mathrm{DH}$ in fear conditioning seem to correspond with current theory (Matus-Amat, 2004; Sanders et al., 2003). Pre-training lesions to the hippocampus reduce acquisition of context fear, but this finding is time limited. Excitotoxic lesions made one week or longer before testing, as were made in the current study, produce no deficits in contextual fear, indicating that other structures can compensate for the hippocampus if hippocampal damage occurs. According to this model, the hippocampus functions to (1) mediate the acquisition \& consolidation of a memory during context conditioning (forms a contextual representation), and (2) inhibits the cortex from forming a redundant contextual representation. If hippocampal damage occurs prior to conditioning, then inhibition is lifted allowing the neocortex to form a contextual representation (Fanselow, 2000). Thus, pre-training lesions to the $\mathrm{DH}$ in the current study likely failed to attenuate contextual fear conditioning during exposure to painful and predatory threat stimuli because the neocortex was able to form a contextual representation during threat exposure. 


\section{CHAPTER 5. METHODS AND PROCEDURES: VENTRAL HIPPOCAMPUS}

\subsection{Animals}

Subjects were 22 male Long-Evans hooded rats born and reared from the breeding colony in Snyder Hall at the University of Hawaii. Animals weighed between 290 and 465 grams at the time of surgery. Following weaning (21 days), all animals were singly housed under controlled temperature (23 C) and illumination (12:12 hour light/dark cycle, with lights on at $0600 \mathrm{~h}$ ) with free access to food and water.

\subsection{Surgery}

Prior to surgery, animals were randomly assigned to either a VH experimental-lesion group ( $\mathrm{N}=15)$, or to a sham operated-control group $(\mathrm{N}=7)$. Subjects were deeply anesthetized with an intraperitoneal injection of sodium pentobarbital ( $65 \mathrm{mg} / \mathrm{kg} \mathrm{IP})$ and were mounted in a stereotaxic apparatus (David Kopf Instruments, Tujunga, CA). The scalp was incised and retracted, and the head was positioned to place Bregma and Lambda in the same horizontal plane. Two sets of small burr holes ( $2.0 \mathrm{~mm}$ in diameter) were drilled in the skull bilaterally in order to place a stainless steel cannula ( $28 \mathrm{G}$, Plastics One) into the VH. The cannula was connected to a $10 \mathrm{ul}$ micro syringe (Hamilton) mounted in an infusion pump (Harvard Apparatus) to control the rate of infusion $(0.04 \mathrm{ul} / \mathrm{min})$. VH-lesioned animals received two separate bilateral infusions $(0.30 \mathrm{ul})$ of a $.1 \%$ ibotenic acid solution (Sigma) in the dorsal and ventral regions of the VH (Paxinos and Watson, 1999): dorsal (-5.2 mm posterior to bregma, +/- $5.3 \mathrm{~mm}$ from the midline and $-6.0 \mathrm{~mm}$ ventral from the surface of the brain); ventral $(-5.2 \mathrm{~mm}$ posterior to bregma, $+/-5.3 \mathrm{~mm}$ from the midline and -7.1 ventral from the surface of the brain). 
Following the infusion, the cannula remained in place for an additional five minutes to ensure complete diffusion of the ibotenic acid. For sham-operated controls, the cannula was placed $0.5 \mathrm{~mm}$ above the $\mathrm{VH}$ and no ibotenic acid was infused. Thus, any differences found between lesion and control animals could not be attributed to cortical damage in the experimental group. Following surgery, animals were returned to their home cage and allowed a one-week recovery time prior to the start of behavioral testing.

\subsection{Behavioral Testing Procedures}

Behavioral testing was conducted between $0900-1300$ hours in the following sequence: Cat Odor Test - Cat Exposure Test - foothock, each beginning on consecutive days. Each of the three behavioral tests measured both unconditioned and conditioned defensive behaviors. Unconditioned defensive behaviors were assessed during (cat odor and cat exposure), or immediately following the exposure to the threat stimulus (footshock). Conditioned defensive behaviors were assessed 24 hours after exposure to the threat stimulus in the same context.

All trials were recorded on videotape for subsequent behavioral analysis from an observer blind to experimental conditions. Between every subject trial, each apparatus was cleaned using a $5 \%$ ethanol solution.

\subsection{Behavioral Measures}

All trials were recorded on videotape for subsequent behavioral analysis from a highly trained observer blind to experimental conditions. The specific dependent measures in each test condition varied, but are listed following the description of each behavioral test. 
The specific behaviors measured included: 1) freezing - complete cessation of movement other than respiration; 2) stretch approach - forward ambulation with flat back and stretched neck; 3) stretch attend - standing on all four paws with flat back and stretched neck; 4) rearing - standing on rear paws with forepaws raised off the ground; 5) standing - both fore and hind limbs extended ; 6) crouching - elevation of the forelimbs off the floor and arching of the back; 7) grooming - movement of forepaws or tongue over the body; 8) sniffing - olfactory investigation evidenced by vertical or lateral head movements; scoring initiated when nose visibly moved more than $1 \mathrm{~cm}$; 9) transits - line crossing between far, medium and near locations; measured as any movement from one marked section of the apparatus to another; 10) Contact - direct contact with the wire mesh or block stimulus; measured as direct paw or head contact; 11) Location - duration of time spent in the near, medium or far compartment relative to the threat stimulus (cat odor: block; cat exposure: wire-mesh divider).

\subsection{Behavioral Tests}

\subsubsection{Cat Odor}

The test apparatus consisted of a white Plexiglas runway $(100 \times 12 \times 50 \mathrm{~cm})$ with a clear Plexiglas front panel to permit observation and videotaping. A cloth-wrapped solid plastic block $(9 \times 9 \times 2 \mathrm{~cm})$ was rubbed for five minutes against the fur of laboratoryhoused domestic male cat for three consecutive days and was then stored in a Ziploc plastic bag until serving as the cat odor stimulus. Both control and experimental animals were habituated to the apparatus for three consecutive days without the presence of an odor block. On the fourth day (unconditioned behavior test), the cat odor block was 
placed at one end of the runway and a subject was placed at the opposite end, facing away from the cat odor block. Twenty-four hours later each subject was retested in the same apparatus without the cat odor stimulus (conditioned behavior test). A clothwrapped solid plastic block never exposed to cat odor served as the cue during the conditioned behavioral test. All sessions were ten minutes in duration and were conducted under red light to ensure the odor stimulus remained as ambiguous as possible.

The test apparatus was divided into three equal sections, far, medium and near the odor stimulus. Measures regarding each subject's location were investigated and included transits between sections and contacts with the odor stimulus (block). Behavioral measures were also examined and included freezing, a composite of the two risk assessment measures (stretch attend and stretch approach), and grooming, head movement, rearing, standing and crouching.

\subsubsection{Cat Exposure}

The test apparatus consisted of two adjacent subject chambers $(50 \times 20 \times 30 \mathrm{~cm})$ separated by an opaque white Plexiglas wall, with a wire-mesh screen separating these chambers and the adjoining cat compartment $(55 \times 40 \times 35 \mathrm{~cm})$. Two subjects were simultaneously placed, one in each subject chamber, facing away from the cat compartment (the same cat used in the cat odor test). Following a five-minute pre-cat exposure period, the cat was placed in the cat compartment for ten-minutes (unconditioned behavior test). Twenty-four hours later, subjects were retested in the same apparatus without the cat stimulus (conditioned behavior test). Each test was conducted under white light to ensure the cat stimulus was as unambiguous as possible. 
The test apparatus was divided into three equal sections, far, medium and near the cat stimulus. Measures regarding each subject's location were investigated and included transits between sections and contacts with the wire-mesh divider. Behavioral measures were also examined and included freezing, a composite of the two risk assessment measures (stretch attend and stretch approach), and grooming, head movement, rearing, standing and crouching.

\subsubsection{Footshock}

The footshock test apparatus $(25 \times 25 \times 50 \mathrm{~cm})$ was constructed of black Plexiglas with a clear front panel to permit observation and videotaping. A scrambled electric foot shock from a LaFayette-82401 generator was delivered via a stainless steel grid floor to serve as the painful threat stimulus. After a three-minute habituation period, three footshocks (1.0 mA, $1 \mathrm{~s}$ duration) were delivered at one-minute intervals, followed by a five-minute observation period (unconditioned behavior test). Twenty-four hours later each subject was retested in the same apparatus without the shock stimulus (conditioned behavior test). Each test was conducted under white light in order to ensure that the shock stimulus remained as unambiguous as possible. Behavioral measures included freezing, grooming, head movement, rearing, standing and crouching.

\subsection{Histology}

Following the completion of behavioral testing, histological verification of lesion placement was performed. All Subjects were overdosed with sodium pentobarbital and were perfused transcardially with $0.9 \%$ saline followed by $10 \%$ formalin. Following 
extraction from the skull, brains were placed into a $10 \%$ formalin solution for 48 hours and then transferred to a $30 \%$ sucrose-formalin solution until blocking (at least 48 hours) and sectioning on a cryostat (Leica). A series of coronal sections were collected (50 um thick taken every $100 \mathrm{um}$ ) and after drying (24 hours), the sections were thionin-stained in order to visualize cell damage to the stained brain sections using a microscope (Leica).

To assess the amount of damage to the $\mathrm{VH}$, sections were mapped on schematic representations employing the rat brain atlas of Swanson (2004).

\subsection{Behavioral Analysis}

Independent Student's t-tests were performed on each dependent measure (unconditioned and conditioned), for both the frequency and duration of each measure. Alpha was set at 0.05 . Mann-Whitney $U$ tests were used to assess the statistical reliability of skewed data. 


\section{CHAPTER 6. RESULTS: VENTRAL HIPPOCAMPUS}

\subsection{Histology}

Figure 2 presents serial histological reconstructions of excitotoxic $\mathrm{VH}$ lesions for rats included in the statistical analyses for each experimental condition. Five lesion animals were excluded from statistical analysis due to partial or complete unilateral sparing of

cells in the VH. All of the rats included the VH experimental groups suffered complete, bilateral lesions of the $\mathrm{VH}$ formation, with minimal damage to surrounding structures. This minimal damage was largely restricted to cannula tract marks and was therefore equivalent to the damage found in the sham-lesioned controls. Nearly all the remaining cells were found in the most posterior ventral and dorsal portions of the VH (CA-2 region). Thus, most of the $\mathrm{VH}$ was destroyed including virtually all of the granular neurons in the dentate gyrus, as well all of the pyramidal cells in regions CA-1 and CA-3.

\subsection{Unconditioned Behavior Results}

\subsection{1. $\underline{\text { Cat Odor }}$}

The frequencies and durations of each location and behavioral measure during the cat odor test for both the VH-lesion and sham-operated control groups are presented in figure 3 and table 4, respectively. With respect to measures of location, lesion and sham animals did not significantly differ in the duration of contact with the odor block, $t(15)=$ $1.51, p>0.05$, or time spent near, medium or far from the stimulus, $t(15)=1.95, p>$ $0.05, t(15)=1.03, p>0.05, t(15)=1.91, p>0.05$, respectively. $\mathrm{VH}$ lesions significantly reduced the duration of freezing, $t(15)=4.01, p<0.005$, while increasing the amount of sniffing $t(15)=4.03, p<0.005$, compared to controls; but had no effect on risk 
assessment, $t(15)=0.16, p>0.05$. Neither group differed on durations of time spent standing, $t(15)=1.70, p>0.05$, or grooming, $t(15)=0.49, p>0.05$, but lesion animals displayed significantly reduced durations of crouching, $t(15)=2.14, p<0.05$, and increased levels of rearing, $t(15)=2.67, p<0.05$, compared to controls. There was no difference in the number of transits between the two groups, $t(15)=2.01, p>0.05$.

\subsubsection{Cat Exposure}

Figure 4 and table 5 present the frequencies and durations of each behavioral and location measure during the cat exposure test for both the VH-lesion and sham-operated controls. With respect to location measures, nether group significantly differed in the duration of contact with the wire mesh, $t(15)=0.90, p>0.05$, however, subjects in the lesion group spent significantly more time near the predatory threat stimulus, $t(15)=$ $2.49, p<0.05$. Neither group spent any time in the medium location relative to the cat stimulus, however, VH lesions significantly reduced the amount of time spent in the far location $t(15)=2.49, p<0.05$. The durations of freezing, $t(15)=0.93, p>0.05$, and sniffing $t(15)=0.91, p>0.05$, were not significantly different, and neither group engaged in risk assessment. There were no significant group differences in durations of standing, $t(15)=1.31, p>0.05$, crouching, $t(15)=0.97, p>0.05$, or rearing, $t(15)=0.83, p>0.05$ and neither group displayed any grooming or engaged in any transits.

\subsubsection{Footshock}

The durations of each behavior during the post footshock test for both the VH-lesion and sham-operated controls are depicted in figure 5 and table 6 , respectively. VH lesions 
significantly reduced the duration of freezing, $t(15)=3.93, p<0.005$, while increasing the amount of sniffing, $t(15)=3.93, p<0.005$, compared to controls. Neither group engaged in grooming and there were no significant differences between groups in the amount of standing, $t(15)=1.03, p>0.05$, crouching, $t(15)=1.87, p>0.05$, or rearing, $t(15)=1.81, p>0.05$

\subsection{Conditioned Behavior Results}

\subsubsection{Cat Odor Cue + Context Conditioning}

Figure 3 and table 4 present the frequencies and durations of each location and behavioral measure during the cat odor test for cue + context conditioning for both the VH-lesion and sham-operated control groups. With respect to measures of location, groups did not significantly differ in the duration of contact with the non-odor block, $t(15)=1.40, p>0.05$, or time spent near, medium or far from the stimulus, $t(15)=1.39$, $p>0.05, t(15)=0.90, p>0.05, t(15)=1.60, p>0.05$, respectively. $\mathrm{VH}$ lesions significantly reduced durations of freezing, $t(15)=2.58, p<0.05$, and risk assessment, $t(15)=2.34, p<0.05$, while increasing the amount of sniffing $t(15)=2.39, p<0.05$, compared to controls. Neither group differed on durations of time spent standing, $t(15)=$ $0.71, p>0.05$, grooming, $t(15)=0.0 .64, p>0.05$, crouching, $t(15)=1.39, p>0.05$, or rearing, $t(15)=1.21, p>0.05$. There was no difference in the number of transits between the two groups, $t(15)=1.42, p>0.05$. 


\subsubsection{Cat Exposure Context Conditioning}

Both the frequencies and durations of each behavioral and location measure during the cat exposure context conditioning test for both the VH-lesion and sham-operated controls are presented in figure 4 and table 5 respectively. For measures of location, neither group significantly differed in the duration of contact with the wire mesh, $t(15)=$ $1.56, p>0.05$, or in the amount of time spent near, medium and far from the threat stimulus, $t(15)=1.03, p>0.05, t(15)=0.85, p>0.05, t(15)=1.15, p>0.05$, respectively. VH lesions significantly reduced durations of freezing, $t(15)=4.16, p<$ 0.001 , and increased the amount of sniffing $t(15)=2.52, p<0.05$, compared to controls, but had no effect risk assessment, $t(15)=0.86, p>0.05$. There were no significant group differences in durations of standing, $t(15)=1.24, p>0.05$, or rearing, $t(15)=0.93, p>$ 0.05 , but VH lesions significantly reduced the duration of crouching, $t(15)=2.20, p<$ 0.05 , and increased the amounts of grooming, $t(15)=2.38, p<0.05$, and transits $t(15)=$ 2.34, $p<0.05$, compared to controls.

\subsubsection{Footshock Context Conditioning}

Figure 5 and table 6 present the durations of each behavior during the footshock context conditioning test for both the VH-lesion and sham-operated controls. VH lesions significantly reduced the duration of freezing, $t(15)=3.39, p<0.005$, and increased the amount of sniffing, $t(15)=3.34, p<0.005$, compared to controls. There were no significant group differences in the amount of standing, $t(15)=1.62, p>0.05$, crouching, $t(15)=1.95, p>0.05$, grooming, $t(15)=0.83, p>0.05$, or rearing, $t(15)=1.44, p>0.05$. 


\section{CHAPTER 7. DISCUSSION: VENTRAL HIPPOCAMPUS}

\subsection{Unconditioned Behaviors}

Results from the current study help to clarify findings from earlier research implicating the hippocampus in the selection of unconditioned defensive behaviors (Blanchard and Blanchard, 1972; Kim et al, 1971; Blanchard and Blanchard, 1970). Findings support the hypothesis that the VH is specifically involved in modulating unconditioned responses to predatory and painful threat stimuli, as $\mathrm{VH}$ lesions reduced certain measures of defense. During the cat odor test, VH lesions reliably reduced durations of freezing and crouching, while increasing levels of rearing and sniffing. During the cat exposure test, animals with $\mathrm{VH}$ lesions spent a greater proportion of time near the wire-mesh separating them from the cat, while controls spent the majority of the test session as far away from the threat stimulus as possible. While this may suggest that VH lesions reduced avoidance of the predator, when the cat was introduced both sham and lesion animals froze in their respective locations, making no transits during the exposure period. Thus, differences in location measures during the exposure period should be interpreted with caution. Finally, during the post footshock test, $\mathrm{VH}$ lesions reliably reduced levels of freezing, while increasing levels of olfactory investigation (sniffing). Collectively, these results indicate that VH lesions reduce certain measures of fear/anxiety (freezing and crouching), while increasing measures of risk assessment (rearing) and olfactory investigation (sniffing) in certain tests of defense.

The general reduction in defensive behaviors seen in the current study following VH lesions can best be understood by examining the testing paradigms in which the behaviors occurred. Testing situations where rats are presented with specific, discrete 
threat stimuli where escape is not possible (cat exposure) represent situations closely related to fear, whereas tests exposing rats to situations of potential or anticipated threat rather than actual immediate danger (cat odor) represent situations similar to anxiety (Blanchard, Blanchard and Rogers, 1990). When normal rats are exposed to an inescapable, discrete predatory threat stimulus (cat), their prominent behavior exhibited is freezing (Blanchard and Blanchard, 1990); and risk assessment will only occur after these initial behaviors subside following the removal of the predator (Blanchard and Blanchard, 2003). However, when rats are exposed to a non-discrete or potential threat stimulus (cat odor), an initial period of immobility (freezing) subsides and is replaced by periods of risk assessment. Following foot-shock, animals tend not to engage in risk assessment as no identifiable threat stimulus is present to attend to, and thus animals generally exhibit initial escape attempts (jump escape) immediately followed by freezing.

In the present study, control animals demonstrated the same pattern of behaviors. During the cat odor test, subjects with VH lesions displayed lower durations of immobile postures (freezing and crouching), which were replaced by more active exploratory behaviors such as rearing and general olfactory investigation (sniffing). It should be noted that rearing represents an additional measure of risk assessment, one that tends to occur in situations with potential or low threat levels (cat odor), following the risk assessment measures of stretch approach and stretch attend, wherein the animal has learned that threat is not immanent (Blanchard and Blanchard, 1990). Thus, during cat odor VH lesions produced a shift from normal freezing and stretch attend postures to more general exploratory behavior, suggesting a role for the $\mathrm{VH}$ in the modulation of unconditioned defense to potential threat stimuli. 
During the cat exposure test $\mathrm{VH}$ lesions failed to produce any decrements in freezing or risk assessment, which suggests that the VH does not modulate defensive reactions during exposure to potent/actual threat stimuli. Thus, during cat odor VH lesions were able to reduce defensiveness to olfactory cues, while the additional auditory and visual cues inherent in the cat stimulus rendered the animals unresponsive to the effects of $\mathrm{VH}$ lesions. A different interpretation of these paradoxical effects of $\mathrm{VH}$ lesions on defense behaviors during cat odor and cat exposure is that the $\mathrm{VH}$ plays a specific role in the selection of defensive behaviors related to tests of anxiety (cat odor), without impacting behaviors related to fear (cat exposure). This conclusion gains support from numerous studies showing that VH lesions produce anxiolytic-like effects in four tests of anxiety, variations of the EPM, SPBT, social interaction and hyponeophagia (Degroot and Treit, 2004; McHugh et al., 2004; Trivedi and Coover, 2004; Bannerman et al., 2003; Kjelstrup et al., 2002). Furthermore, the anxiolytic-like effects seen in these studies resulting from VH lesions resemble the anxiolytic effects induced by benzodiazepines on many of the same behavioral tests (Gray and McNaughton, 2000; Menard and Treit, 1999).

Lastly, it is difficult to argue that behaviors observed following footshock represent anxiety or fear-like behaviors. In this testing situation, the animal is not capable of avoiding or assessing the risk associated with the threat source. However, the animal clearly learns to "fear" the chamber/environment associated with even a single shock (Blanchard and Blanchard, 1969), evidenced by both conditioned post footshock freezing and contextual freezing twenty-four hours following the conditioning session in the absence of any footshocks. Thus, one could argue that post footshock freezing represents anticipatory fear (Sanders et al., 2003). However, post footshock behavioral responses 
could also be described as representing anxiety-like responses because anxiety involves the modulation of preexisting fear (McNaughton and Corr, 2004). In either respect, VH lesions reduced defensive behaviors measured by a reduction in immobility (freezing \& crouching) and an increase in olfactory investigation (sniffing), indicating that the $\mathrm{VH}$ is involved in the neural system or systems controlling the elicitation of innate defensive responses to painful threat stimuli.

A possible mechanism by which the ventral, not dorsal hippocampus serves to modulate the expression of defense behaviors is through VH connections with the hypothalamus and amygdalar complex. CA1 regions of the $\mathrm{VH}$ and ventral subiculum project to the LSrvld (Petrovich et al., 2001; Risold \& Swanson, 1996), which projects to AHN and PMd of the MHZ defensive system. Additionally, CA1 regions of the VH, ventral subiculum and entorhinal cortex have bilateral projections with the amygdalar complex, including the lateral (LA), basolateral (BL), basomedial (BM) and medial (MeA) nuclei (Petrovich et al., 2001; Pikkarainen, et al., 1999; Canteras \& Swanson, 1992a), structures previously implicated in defensive behavior. The MeA receives dense input from both the main and accessory olfactory systems (Rosen, 2004) and has high levels of c-fos expression during cat odor exposure (Dielenberg et al., 2001). Lesions restricted to the MeA reduced freezing and risk assessment during cat odor exposure $(\mathrm{Li}$, Maglinao and Takahashi, 2004; Markham et al., unpublished data), while having no effect on freezing levels during cat exposure or post footshock. These findings suggest that the reductions in anxiety-like behaviors produced by VH lesions during cat odor could result from ventral hippocampal modulation of the defensive circuit via projections through the MeA. While the current study also found a reduction in defensiveness 
following footshock, this may have resulted from ventral hippocampal projections through the $\mathrm{LA}, \mathrm{BL}$ or $\mathrm{BM}$ innervating the $\mathrm{MHZ}$ defensive system. In support of this assertion, Rosen (2004) found that neurotoxic lesions of the basolateral complex, including the LA and BL nuclei, reduced defensiveness during a post footshock freezing test. Thus, the ventral and not dorsal hippocampus may help organize the selection of defensive responses through its projections with the MHZ defensive circuit via amygdalar and/or septal nuclei.

A possible argument against the notion that the $\mathrm{VH}$ plays a significant role in modulating innate unconditioned defensive responses is that hippocampal lesions simply produce non-specific effects on general activity. That is, hippocampal lesions produce deficits in movement arrest (Blanchard et al., 1977), or directly increase locomotor activity, thus rendering the animal incapable of freezing (McNish, Gewirtz, and Davis, 1997). This conclusion is often based on lesion studies that demonstrate an increase in locomotion following hippocampal lesions (Maren and Fanselow, 1997; McNish et al., 1997; Blanchard and Blanchard, 1972). However, these studies generally utilize electrolytic lesion techniques, which likely produce hyperactivity resulting from damage to fibers of passage. Accordingly, similar increases in locomotion or hyperactivity were not detected in numerous studies that demonstrated similar anxiolytic-like effects, utilizing either excitotoxic (Degroot and Treit, 2004; Trivedi and Coover, 2004; Bannerman et al., 2003; Kjelstrup et al., 2002) or reversible lesion (Bast and Feldon, 2003; Bast et al., 2001) techniques. Methods of detecting anxiolytic-like effects in both the EPM and SPBT attained by either increasing hippocampal levels of acetylcholine (Degroot and Treit, 2002), or temporarily inactivating the VH (Degroot and Treit, 2004) 
also contradict this argument. In order for an animal to demonstrate a reduction in anxiety-like behavior in the EPM, an increase in general activity must occur, resulting in increased open arm exploration. Conversely, during the SPBT an anxiolytic-like effect requires a reduction in general activity, measured by a decrease in burying behavior. Thus, the concurrent reduction in anxiety measured by these two tests makes it difficult to conclude that the effects of $\mathrm{VH}$ lesions in the current study resulted from non-specific effects on general activity (Degroot and Treit, 2002).

Results from the current study provide additional evidence against a general activity argument for the role of the $\mathrm{VH}$ in defensive behavior elicitation. If $\mathrm{VH}$ lesions simply render animals incapable of movement arrest, then animals with VH lesions should not be able to maintain a freezing posture. Clearly, the results from the cat exposure test argue against this notion since $\mathrm{VH}$ lesion and sham operated controls did not differ on any measure of activity, with both groups freezing for almost the entire duration of the test (percent freezing: $\mathrm{VH}=93 \%$, sham $=99 \%$ ). Thus, when presented with a potent, discrete fear producing threat stimulus, VH lesioned animals are capable of demonstrating the appropriate fear response, complete immobility (ten-minute duration). Furthermore, results from chemical manipulation studies suggest that stimulation of the VH increases locomotion, while temporary inactivation decreasing locomotor activity (Bast and Feldon, 2003). This finding is inconsistent with the explanation that VH lesions in the current study, which abolished VH functioning, simply resulted in increased levels of locomotion. Collectively, results from the current study and previous studies employing excitotoxic stimulation, deactivation or lesion techniques, suggest that the reduction in unconditioned defensive behaviors seen in the current study cannot be 
attributed to a general increase in locomotion. A different and more likely interpretation is that one function of the $\mathrm{VH}$ is to modulate active defensive behaviors by providing inhibitory and excitatory input into the MHZ defensive circuit via septal and amygdaloid nuclei (Canteras, 2002).

\subsection{Conditioned Behaviors}

In addition to suggesting a role during the selection of unconditioned defensive behaviors, results from the current study also provide evidence that the VH is involved in the expression of conditioned defensive behaviors during exposure to both predatory and painful threat stimuli. During the cat odor cue + context conditioning test, $\mathrm{VH}$ lesions significantly reduced levels of freezing and the composite measure of risk assessment (stretch approach and stretch attend), while increasing levels of olfactory investigation (sniffing). Likewise, during the contextual conditioning test in the cat exposure apparatus, VH lesions significantly reduced durations of freezing and crouching, while increasing the durations of olfactory investigation (sniffing) and grooming, and the number of transits. Lastly, during the contextual conditioning test twenty-four hours following footshock, lesioned subjects displayed a significant reduction in freezing, while engaging in higher durations of olfactory investigation (sniffing), compared to controls.

The similar reductions in conditioning measures detected in both the cat odor and cat exposure-conditioning paradigms, suggest that in normal rats, the VH helps modulate the expression of conditioned defensive behaviors in animal models related to fear and anxiety. Projections from the MHZ defensive system to the hippocampal formation provide a possible explanation for the role of the $\mathrm{VH}$ in these conditioning paradigms. 
The nucleus reunions in the dorsal thalamus, receives dense projections from the $\mathrm{AHN}$, VMHdm and PMd (Comoli et al., 2000; Canteras, Simerly and Swanson, 1994; Risold, Canteras and Swanson, 1994; Canteras and Swanson, 1992b), which then projects to the hippocampus via the entorhinal cortex (Wouterlood, Saldana and Witter, 1990). Thus, feedback from this pathway could involve hippocampal functioning during emotional conditioning (Canteras, 2002). Additionally, projections from the VH to the amygdalar nuclei provide an additional neural mechanism by which the VH could modulate conditioned defensive behaviors. Specific amygdalar nuclei targeted by the VH have been implicated in conditioned defensiveness to context as lesions to various components of the amygdala abolish the expression of conditioned fear (Koo, Han and Kim, 2004; Rosen, 2004; Sacchetti et al, 1999; Phillips and LeDoux, 1992; Blanchard and Blanchard, 1972b). Neurotoxic lesions of the lateral (Rosen, 2004) and basolateral nuclei (Koo et al., 2004) abolish conditioned fear (freezing) when shock is used as the conditioning stimulus, while ibotenic acid lesions of the MeA reduce defensiveness to conditioned predatory threat stimuli (Markham et al., unpublished data). Thus, the ventral pole of the hippocampus may operate as part of a neural circuit or circuits subserving the elicitation of defensive behavior through bidirectional connections with various nuclei of the amygdala and hypothalamus.

A second interpretation of the cat exposure contextual conditioning test is that it represents a test of anxiety-like behaviors. This interpretation is possible because the threat stimulus (context) represents a situation in which a fear-evoking stimulus (predator) was previously encountered (Blanchard et al., 1990). Prior to predator exposure, the rat is placed into the apparatus for a five-minute pre-cat exposure period, 
after which the cat is placed into the adjoining chamber for ten minutes. Thus, during the contextual conditioning test (twenty-four hours later) it is reasonable to assume that subjects anticipate encountering the predator. As such, the contextual conditioning test can be considered anxiogenic as anxiety represents the modulation of preexisting fear (McNaughton and Corr, 2004). Given this interpretation, the decrease in initial fear (freezing) followed by an increase in general exploration (sniffing and transits) is in agreement with prior research that demonstrated a reduction in anxiety-like behavior with VH lesions (Degroot and Treit, 2004; McHugh et al., 2004; Bannerman et al., 2003; Kjelstrup et al., 2002), as well as the current results from the cat odor test for unconditioned defensive behaviors.

The reduction in defensive behavior (freezing) observed twenty-four hours after footshock, suggests that ventral, not dorsal hippocampal lesions also modulate the expression of conditioned defensive behaviors related to painful threat stimuli. This notion is in accord with studies demonstrating reduced conditioned fear expression (freezing) to both tone and context using electrolytic (Trivedi and Coover, 2004) and tetrodotoxin reversible lesions (Bast et al., 2001), as well as NMDA receptor antagonists (Zang et al., 2001) and permanent NMDA lesions (Richmond et al., 1999). Collectively, these results suggest that the $\mathrm{VH}$ not only participates in regulating the elicitation of unconditioned defensive behavior, but also functions as part of a neural circuit or circuits controlling the expression of conditioned defensiveness related to both predatory and painful threat stimuli. 


\section{CHAPTER 8. DISCUSSION: GENERAL}

The results from these two studies provide support for the role of the ventral, not dorsal hippocampus in modulating the selection of unconditioned defensive behaviors in testing paradigms more closely related to anxiety (cat odor) and pain (foot-shock), rather than fear (cat exposure). These results also extend findings from previous research implicating the $\mathrm{VH}$ in modulating anxiety-like responses in several variations of the plusmaze, SPBT, social interaction and hyponeophagia tests to more natural models of defense (cat odor). Furthermore, results from the present study fit with the proposed twoprocess model of contextual fear conditioning regarding the role of the $\mathrm{DH}$, and suggest a possible role for the $\mathrm{VH}$ in contextual fear conditioning paradigms that utilize both painful and natural predatory threat stimuli as the conditioning stimulus. Lastly, the results of the present study provide additional support for the proposed MHZ defensive circuit, suggesting that the $\mathrm{VH}$ serves as an upstream structure functioning to modulate key structures of the circuit including portions of the hypothalamus and amygdala.

The current results implicating the VH in modulating anxiety-like responses to both painful and predatory threat stimuli is of particular importance when considering human psychopathology. According to McNaughton and Gray (2000), all clinically effective anxiolytic compounds disrupt septo-hippocampal functioning by disrupting hippocampal theta activity. The similar reduction in anxiety-like behavior produced by VH lesions in the current study suggests that anxiolytic compounds could produce their effects from either direct or indirect interactions with the VH.

Future research investigating the function of the hippocampus in both unconditioned and conditioned defensive behaviors to both predatory and painful threat stimuli should 
incorporate the testing procedures used by Matus-Amat et al. (2004). Temporarily inactivating the dorsal and ventral hippocampus prior to and immediately following context pre-exposure, prior to and immediately following threat stimulus exposure, and immediately before the conditioning test, will allow for a more thorough assessment of the role the dorsal and ventral hippocampus play in the selection of unconditioned and conditioned defensive behaviors, during exposure to predatory and painful threat stimuli. Furthermore, research utilizing these testing procedures should also investigate the possible contributions of specific divisions within the two poles of the hippocampus by selectively lesioning the $\mathrm{CA} 1$ and $\mathrm{CA} 3$ regions of the dorsal and ventral hippocampus. 
TABLE 1.

Cat Odor Exposure Cue + Context Conditioning

\begin{tabular}{lcccc}
\hline Location Measures & Sham Lesions & DH Lesions & Sham Lesions & DH Lesions \\
\hline Far (s) & $554.35 \pm 9.57$ & $548.67 \pm 17.79$ & $499.58 \pm 20.55$ & $525.59 \pm 25.07$ \\
Medium (s) & $16.70 \pm 3.60$ & $10.75 \pm 3.53$ & $33.76 \pm 6.37$ & $22.66 \pm 9.24$ \\
Near (s) & $27.52 \pm 6.75$ & $38.82 \pm 13.97$ & $65.78 \pm 15.14$ & $51.35 \pm 18.10$ \\
Contact (s) & $0.12 \pm 0.12$ & $0.74 \pm 0.50$ & $28.08 \pm 8.24$ & $20.62 \pm 8.30$ \\
\hline Behavioral Measures & & & & \\
\hline Freezing (s) & $366.33 \pm 25.76$ & $338.97 \pm 38.85$ & $200.26 \pm 30.66$ & $149.59 \pm 31.92$ \\
Rear (s) & $64.96 \pm 12.09$ & $49.97 \pm 9.73$ & $181.51 \pm 24.40$ & $157.33 \pm 25.25$ \\
Stand (s) & $309.63 \pm 33.59$ & $272.38 \pm 36.03$ & $218.62 \pm 22.95$ & $246.74 \pm 28.08$ \\
Crouch (s) & $147.40 \pm 32.42$ & $181.78 \pm 47.67$ & $66.17 \pm 22.82$ & $69.71 \pm 27.09$ \\
Head Movement (s) & $153.96 \pm 15.02$ & $160.16 \pm 18.06$ & $259.70 \pm 19.21$ & $318.66 \pm 23.85$ \\
Groom (s) & $23.10 \pm 8.48$ & $46.35 \pm 22.56$ & $48.59 \pm 12.16$ & $52.39 \pm 15.94$ \\
Risk Assessment (s) & $14.40 \pm 2.91$ & $10.19 \pm 3.27$ & $0.69 \pm 0.31$ & $2.55 \pm 1.52$ \\
Transits & $12.07 \pm 2.38$ & $12.40 \pm 3.61$ & $27.00 \pm 3.75$ & $21.60 \pm 7.77$ \\
\hline
\end{tabular}

Effects of DH lesions (mean + S.E.M.) in the cat odor test.

TABLE 2.

Cat Exposure

Context Conditioning

\begin{tabular}{lcccc}
\hline Location Measures & Sham Lesions & DH Lesions & Sham Lesions & DH Lesions \\
\hline Far (s) & $279.84 \pm 79.95$ & $446.78 \pm 81.24$ & $264.16 \pm 66.06$ & $260.11 \pm 73.77$ \\
Medium (s) & $79.96 \pm 54.48$ & $0.03 \pm 0.03$ & $6.61 \pm 1.83$ & $24.19 \pm 18.63$ \\
Near (s) & $199.90 \pm 75.56$ & $152.85 \pm 81.24$ & $326.38 \pm 64.79$ & $306.84 \pm 76.61$ \\
Contact (s) & $39.98 \pm 39.98$ & $0.00 \pm 0.00$ & $2.05 \pm 1.01$ & $8.50 \pm 4.85$ \\
\hline Behavioral Measures & & & & \\
\hline Freezing (s) & $589.88 \pm 7.13$ & $591.35 \pm 5.63$ & $404.17 \pm 30.58$ & $361.22 \pm 55.03$ \\
Rear (s) & $41.02 \pm 39.93$ & $0.00 \pm 0.00$ & $43.30 \pm 15.36$ & $64.11 \pm 24.73$ \\
Stand (s) & $261.70 \pm 76.76$ & $300.30 \pm 85.75$ & $405.15 \pm 47.09$ & $364.88 \pm 53.84$ \\
Crouch (s) & $295.94 \pm 77.40$ & $298.59 \pm 85.97$ & $115.83 \pm 46.09$ & $123.43 \pm 60.32$ \\
Head Movement (s) & $9.80 \pm 6.85$ & $7.68 \pm 5.66$ & $156.68 \pm 23.69$ & $188.11 \pm 38.73$ \\
Groom (s) & $0.04 \pm 0.04$ & $0.00 \pm 0.00$ & $8.42 \pm 3.61$ & $7.87 \pm 3.84$ \\
Risk Assessment (s) & $0.00 \pm 0.00$ & $0.00 \pm 0.00$ & $2.23 \pm 1.60$ & $5.23 \pm 3.23$ \\
Transits & $0.93 \pm 0.07$ & $1.20 \pm 0.20$ & $7.73 \pm 1.45$ & $11.70 \pm 3.77$ \\
\hline
\end{tabular}

Effects of DH lesions (mean + S.E.M.) in the cat exposure test. 
TABLE 3.

Post Footshock Context Conditioning

\begin{tabular}{lcccc}
\hline Behavioral Measures & Sham Lesions & DH Lesions & Sham Lesions & DH Lesions \\
\hline Freezing (s) & $236.92 \pm 13.30$ & $215.71 \pm 26.22$ & $159.16 \pm 21.83$ & $183.73 \pm 18.65$ \\
Rear (s) & $44.53 \pm 20.54$ & $45.46 \pm 28.59$ & $25.15 \pm 8.79$ & $10.09 \pm 4.64$ \\
Stand (s) & $249.42 \pm 20.32$ & $237.84 \pm 28.37$ & $253.06 \pm 19.55$ & $250.00 \pm 21.89$ \\
Crouch (s) & $0.56 \pm 0.46$ & $2.36 \pm 2.22$ & $13.27 \pm 13.27$ & $28.19 \pm 22.80$ \\
Head Movement (s) & $56.84 \pm 11.86$ & $68.67 \pm 19.97$ & $131.16 \pm 20.53$ & $102.95 \pm 14.82$ \\
Groom (s) & $0.00 \pm 0.00$ & $1.78 \pm 1.78$ & $0.00 \pm 0.00$ & $3.48 \pm 2.38$ \\
\hline
\end{tabular}

Effects of DH lesions (mean + S.E.M.) in the shock test.

TABLE 4.

Cat Odor Exposure Cue + Context Conditioning

\begin{tabular}{lcccc}
\hline Location Measures & Sham Lesions & VH Lesions & Sham Lesions & VH Lesions \\
\hline Far (s) & $557.92 \pm 8.90$ & $510.21 \pm 19.78$ & $449.27 \pm 33.65$ & $343.55 \pm 49.68$ \\
Medium (s) & $14.91 \pm 5.06$ & $22.85 \pm 5.41$ & $34.46 \pm 8.14$ & $45.13 \pm 8.11$ \\
Near (s) & $26.96 \pm 4.43$ & $61.75 \pm 14.46$ & $114.35 \pm 25.62$ & $210.38 \pm 54.62$ \\
Contact (s) & $7.49 \pm 2.88$ & $22.46 \pm 8.00$ & $57.15 \pm 13.25$ & $147.34 \pm 52.55$ \\
\hline Behavioral Measures & & & & \\
\hline Rear (s) & $31.25 \pm 8.80$ & $95.33 \pm 18.95^{*}$ & $135.68 \pm 25.14$ & $173.85 \pm 19.68$ \\
Stand (s) & $124.21 \pm 28.03$ & $188.27 \pm 24.73$ & $203.83 \pm 33.76$ & $225.21 \pm 9.61$ \\
Crouch (s) & $393.00 \pm 45.48$ & $236.88 \pm 51.94^{*}$ & $147.33 \pm 41.95$ & $88.18 \pm 20.75$ \\
Head Movement (s) & $204.45 \pm 21.56$ & $363.45 \pm 29.22^{* *}$ & $322.05 \pm 29.30$ & $398.00 \pm 17.16^{*}$ \\
Groom (s) & $24.38 \pm 10.68$ & $30.91 \pm 8.22$ & $49.20 \pm 13.31$ & $41.47 \pm 4.19$ \\
Risk Assessment (s) & $5.19 \pm 2.28$ & $4.65 \pm 2.41$ & $3.01 \pm 1.42$ & $0.20 \pm .20^{*}$ \\
\hline
\end{tabular}

Table 4. Effects of VH lesions (mean + S.E.M.) in the cat odor test, differences for which ${ }^{*} \mathrm{P}<0.05$; differences for which $* * \mathrm{P}<0.01$. 
TABLE 5.

Cat Exposure

Context Conditioning

\begin{tabular}{lrccc}
\hline Location Measures & Sham Lesions & \multicolumn{1}{c}{ VH Lesions } & \multicolumn{1}{c}{ Sham Lesions } & \multicolumn{1}{c}{ VH Lesions } \\
\hline Far (s) & $599.85 \pm 0.14$ & $299.98 \pm 100.00^{*}$ & $309.48 \pm 107.41$ & $176.58 \pm 61.89$ \\
Medium (s) & $0.00 \pm 0.00$ & $0.00 \pm 0.00$ & $54.36 \pm 45.95$ & $21.64 \pm 5.21$ \\
Near (s) & $0.00 \pm 0.00$ & $300.00 \pm 100.00^{*}$ & $236.15 \pm 95.91$ & $353.51 \pm 68.39$ \\
Contact (s) & $0.00 \pm 0.00$ & $5.65 \pm 5.18$ & $7.99 \pm 4.30$ & $25.09 \pm 8.58$ \\
\hline Behavioral Measures & & & & \\
\hline Rear (s) & $0.00 \pm 0.00$ & $3.56 \pm 3.56$ & $39.97 \pm 18.72$ & $61.51 \pm 14.22$ \\
Stand (s) & $0.00 \pm 0.00$ & $3.42 \pm 2.16$ & $141.48 \pm 57.40$ & $220.62 \pm 35.72$ \\
Crouch (s) & $599.85 \pm 0.14$ & $591.54 \pm 7.11$ & $393.44 \pm 78.13$ & $209.97 \pm 44.01^{*}$ \\
Head Movement (s) & $0.17 \pm 0.17$ & $36.54 \pm 33.12$ & $164.27 \pm 47.91$ & $288.78 \pm 24.49^{*}$ \\
Groom (s) & $0.00 \pm 0.00$ & $0.00 \pm 0.00$ & $8.35 \pm 8.35$ & $36.86 \pm 8.11^{*}$ \\
Risk Assessment (s) & $0.00 \pm 0.00$ & $0.00 \pm 0.00$ & $0.00 \pm 0.00$ & $4.03 \pm 3.87$ \\
\hline
\end{tabular}

Table 5. Effects of VH lesions (mean + S.E.M.) in the cat exposure test, differences for which $* \mathrm{P}<0.05$; differences for which $* * \mathrm{P}<0.01$.

TABLE 6.

Post Footshock

Context Conditioning

\begin{tabular}{lcccc}
\hline Behavioral Measures & Sham Lesions & VH Lesions & Sham Lesions & VH Lesions \\
\hline Rear (s) & $11.35 \pm 5.41$ & $52.60 \pm 18.53$ & $8.96 \pm 4.31$ & $31.63 \pm 12.69$ \\
Stand (s) & $38.50 \pm 25.47$ & $71.30 \pm 20.01$ & $45.40 \pm 26.68$ & $113.41 \pm 29.59$ \\
Crouch (s) & $249.40 \pm 29.92$ & $174.45 \pm 26.13$ & $243.48 \pm 27.32$ & $147.63 \pm 36.14$ \\
Head Movement (s) & $35.44 \pm 14.35$ & $122.88 \pm 15.64^{* *}$ & $88.79 \pm 25.80$ & $193.20 \pm 19.02^{* *}$ \\
Groom (s) & $0.00 \pm 0.00$ & $0.00 \pm 0.00$ & $0.00 \pm 0.00$ & $1.79 \pm 1.79$ \\
\hline
\end{tabular}

Table 6. Effects of VH lesions (mean + S.E.M.) in the shock test,

differences for which $* * \mathrm{P}<0.01$. 


\section{FIGURE 1.}
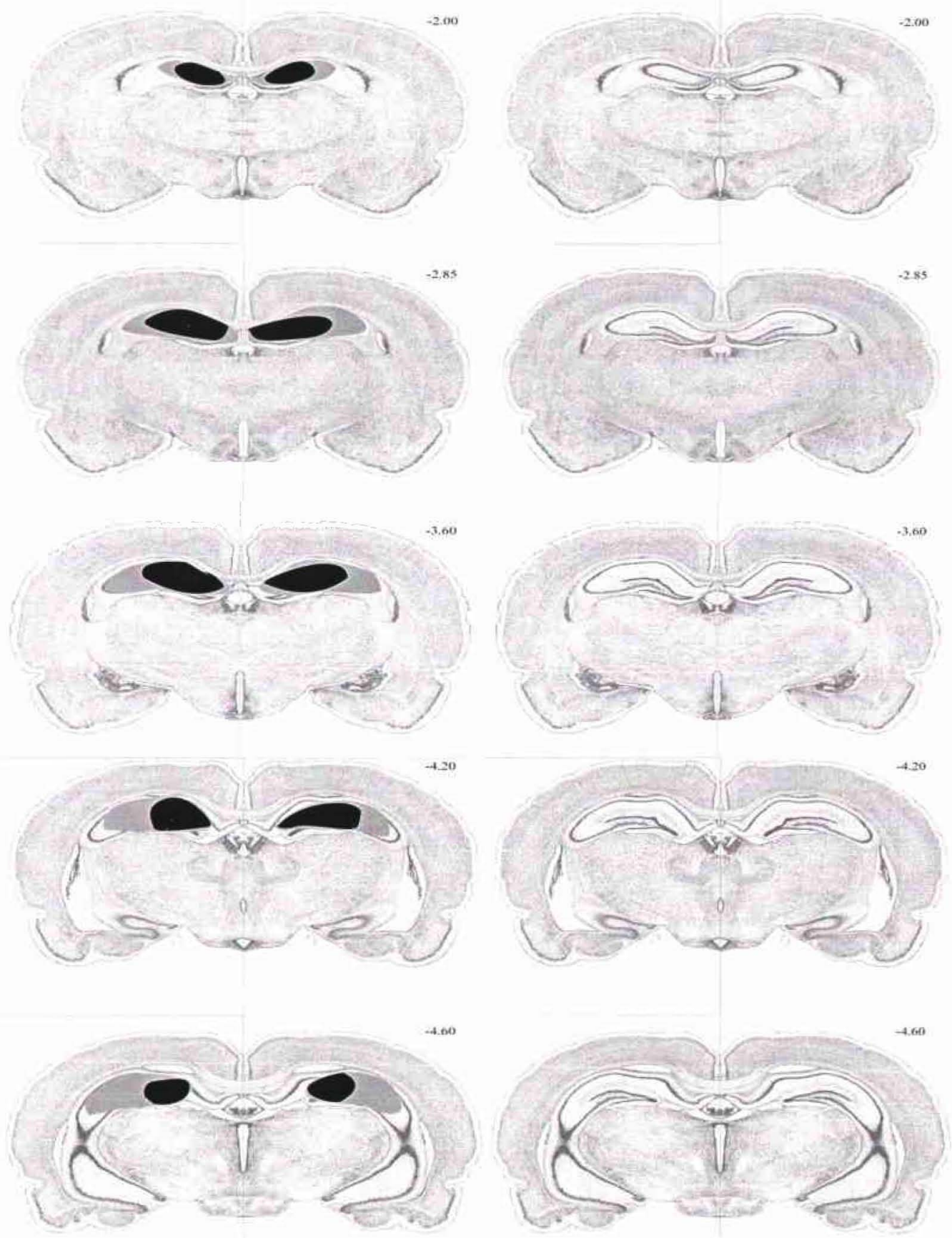

Figure 1. Serial histological reconstructions depicting maximal (grey) and minimal (black) damage to DH (left) and sham (right) lesion animals. 


\section{FIGURE 2.}
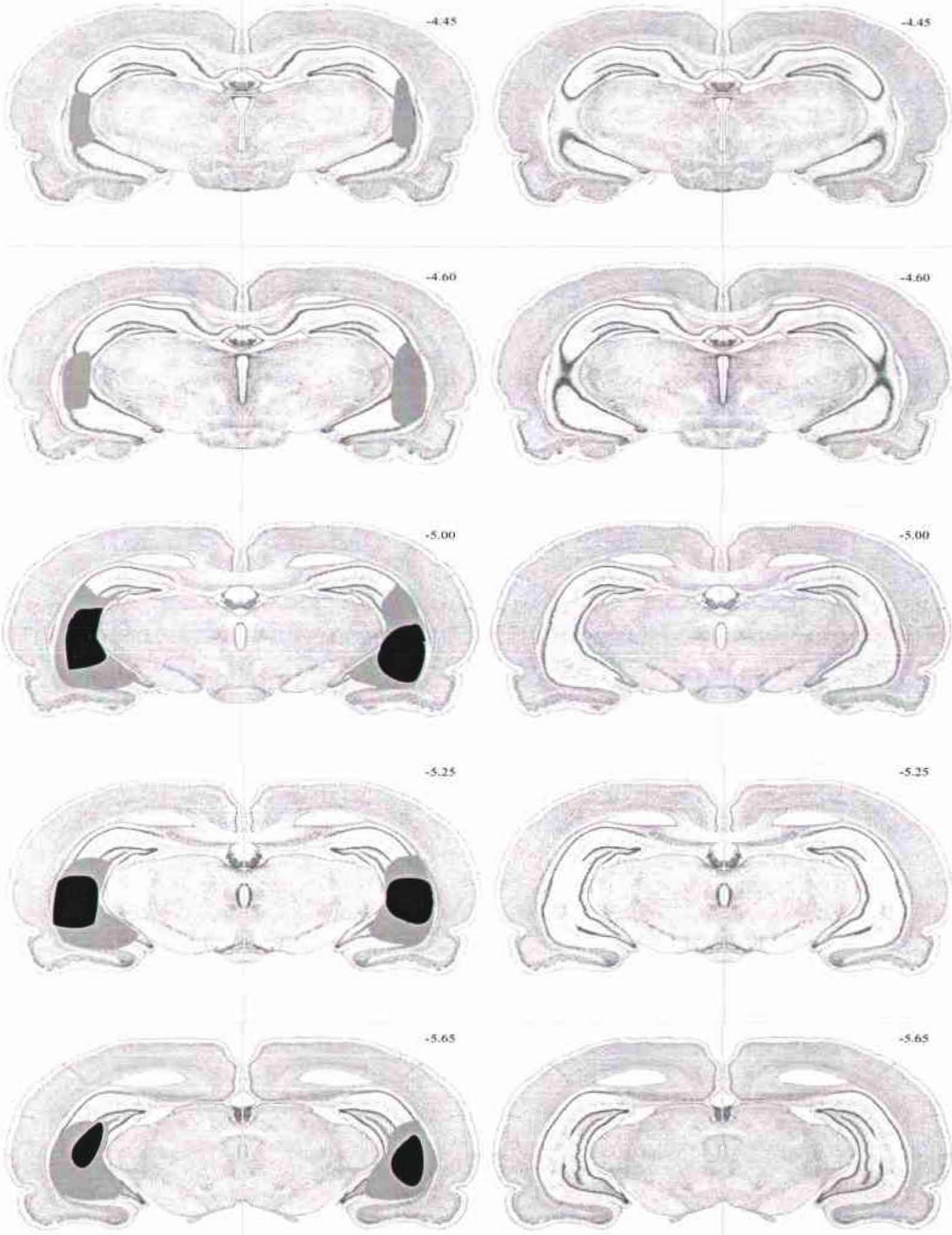

Figure 1. Serial histological reconstructions depicting maximal (grey) and minimal (black) damage to DH (left) and sham (right) lesion animals. 


\section{FIGURE 3.}

\section{Cat Odor Exposure}
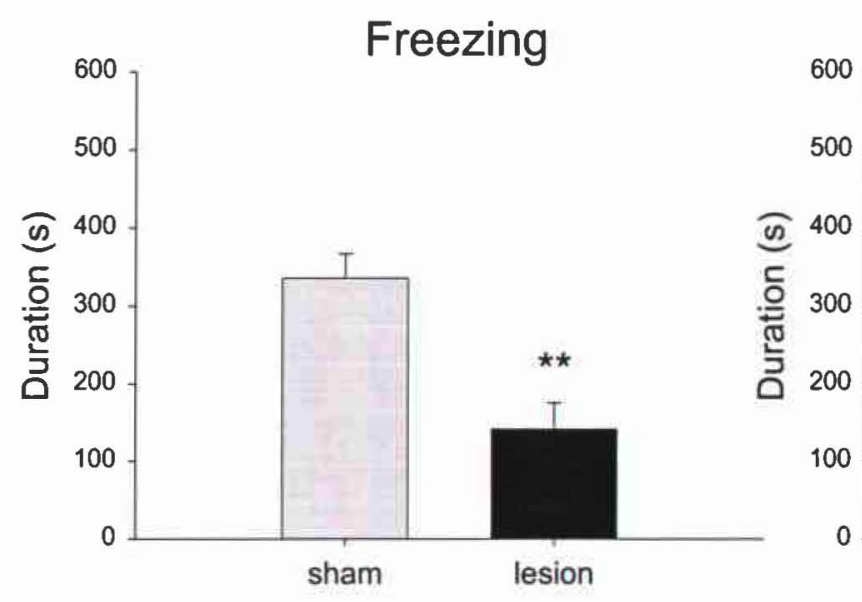

Cue + Context Conditioning
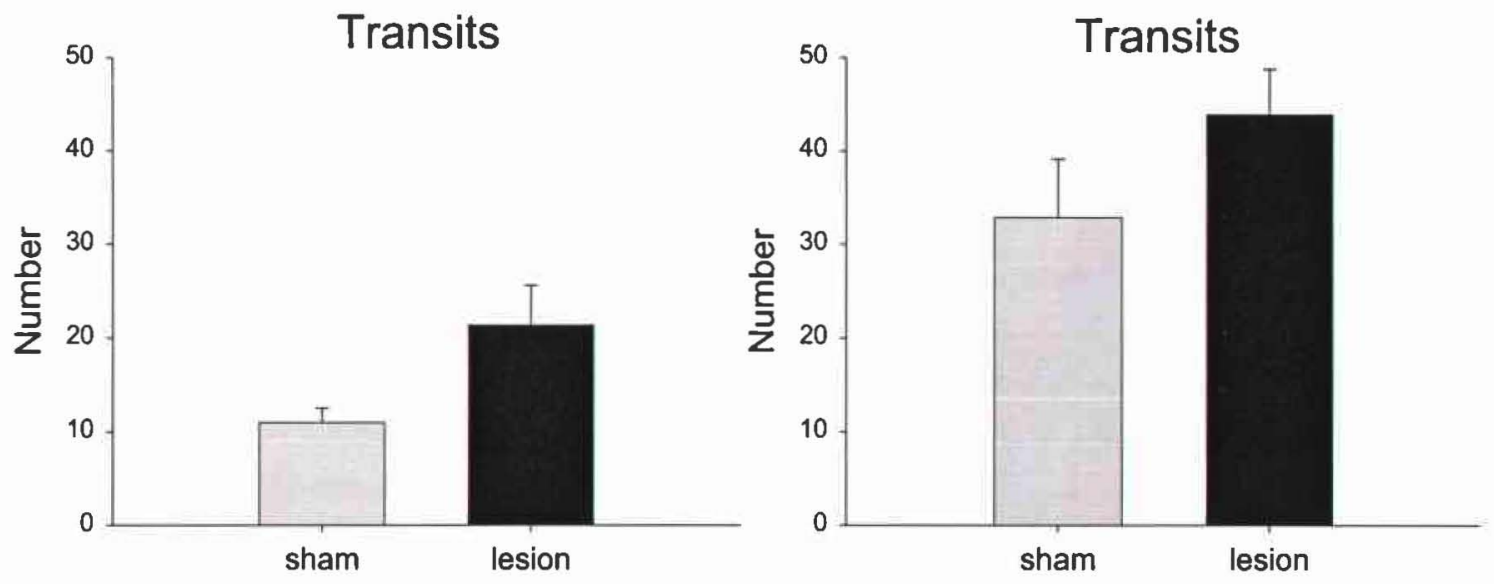

Figure 1. Effects of VH lesions (mean + S.E.M.) in the cat odor test, differences for which ${ }^{*} \mathrm{P}<0.05$; differences for which ${ }^{* *} P<0.01$. 


\section{FIGURE 4}

Cat Exposure

Freezing

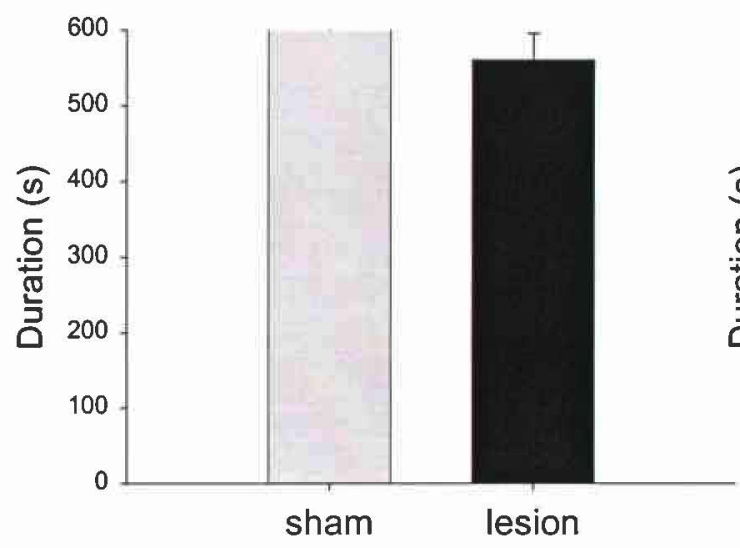

Transits

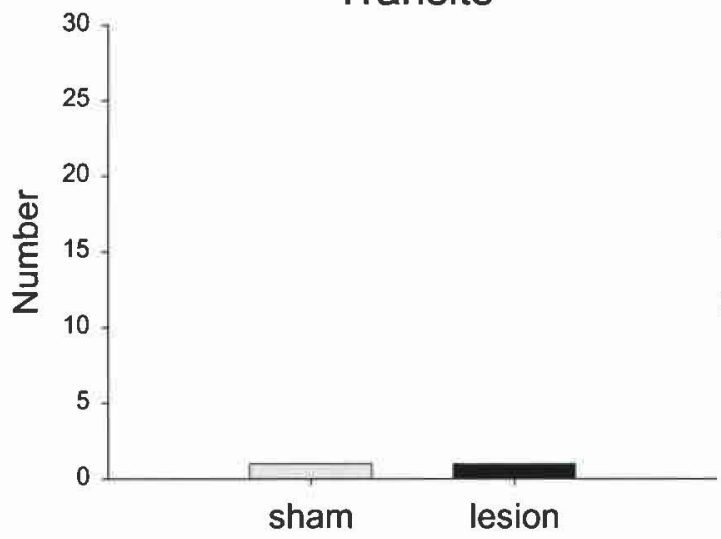

Context Conditioning

Freezing
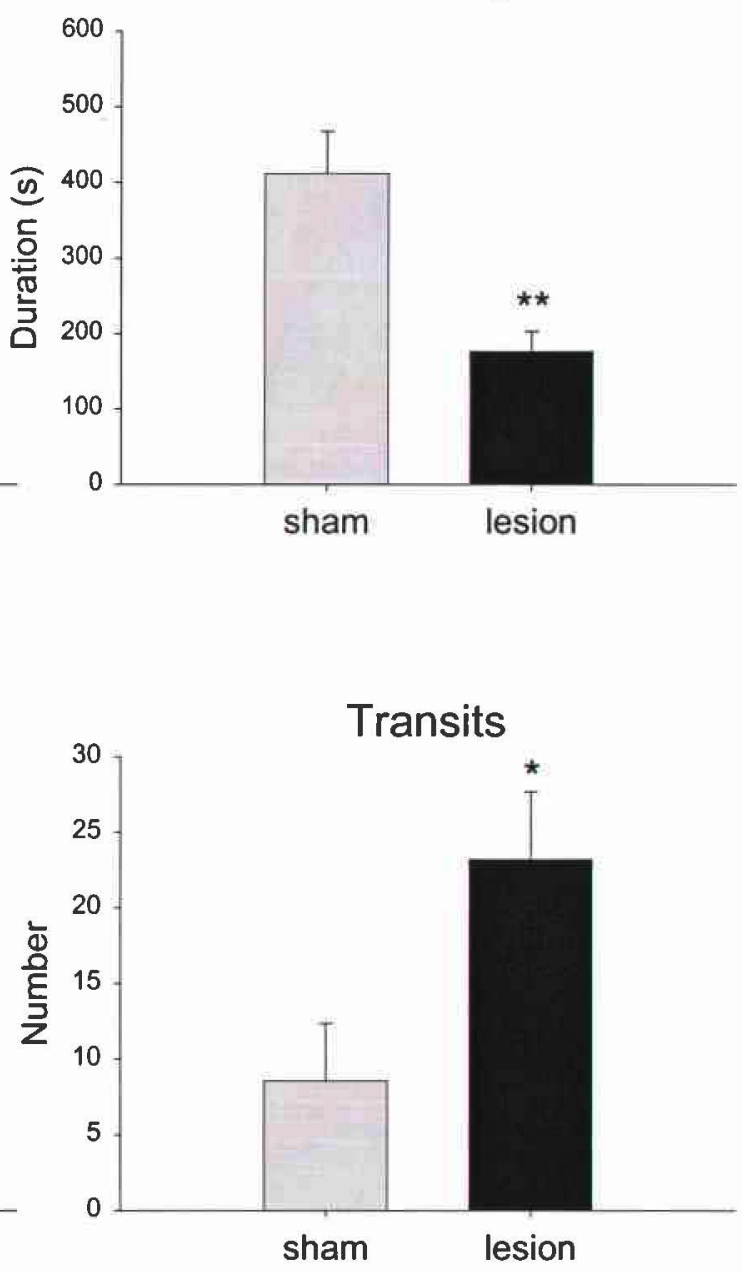

Figure 1. Effects of VH lesions (mean + S.E.M.) in the cat exposure test, differences for which ${ }^{\star} P<0.05$; differences for which ${ }^{\star \star} P<0.01$. 


\section{FIGURE 5.}

Post Shock

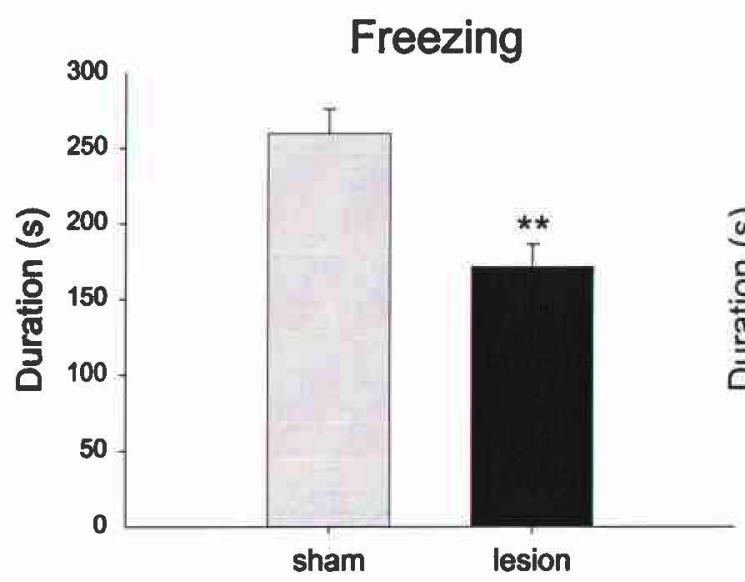

Context Conditioning

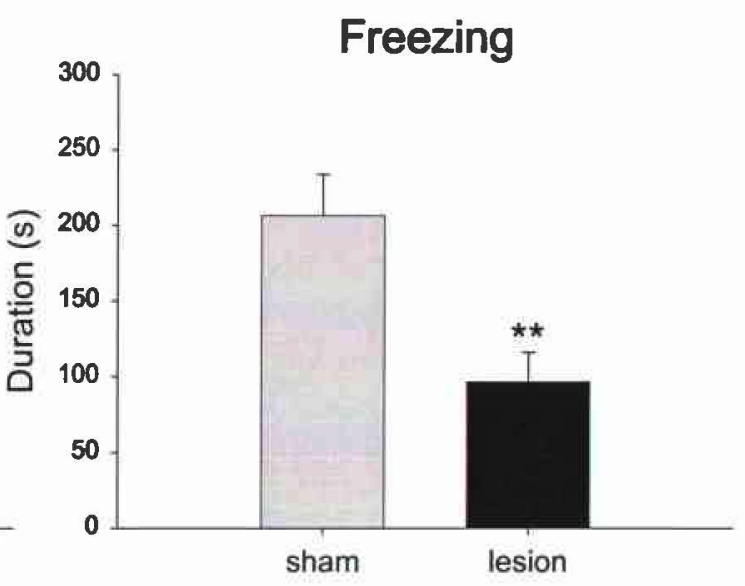

Figure 1. Effects of VH lesions (mean + S.E.M.) in the shock test, differences for which ${ }^{* \pm P}<0.01$. 


\section{REFERENCES}

Albert, D.J., and Chew, G.L. (1980). The septal forebrain and the inhibitory modulation of attack and defense in the rat. A review. Behavior Neural Biology, 30, 357-388.

Anagnostaras, S.G., Gale, G.D., and Fanselow, M.S. (2001). Hippocampus and contextual fear conditioning: Recent controversies and advances. Hippocampus, $11,8-17$.

Bannerman, D.M., Deacon, R.M., Offen, S., Friswell, J., and Grubb, M., Rawlins, J.N. (2002).Double dissociation of function within the hippocampus: spatial memory and hyponeophagia. Behavioral Neuroscience, 116, 884-901.

Bannerman, D.M., Grubb, M., Deacon, R.M.J., Yee, B.K., Feldon, J., and Rawlins, J.N.P. (2003). Ventral hippocampal lesions affect anxiety but not spatial learning. Behavioral Brain Research, 139, 197-213.

Bannerman, D.M., Rawlins, J.N.P., McHugh, S.B., Deacon, R.M.J., Yee, B.K., Bast, T., Zhang, W.-N., Pothuizen, H.H.J., and Feldon, J. (2004). Regional dissociations within the hippocampus-memory and anxiety. Neuroscience and Biobehavioral Reviews, 28, 273-283.

Barrientos, R.M., O’Reilly, R.C., and Rudy, J.W. (2002). Memory for context is impaired by injecting anisomycin into dorsal hippocampus following context exploration. Behavioral Brain Research, 134, 299-306.

Bast, T., and Feldon, J. (2003). Hippocampal modulation of sensorimotor processes. Progress in Neurobiology, 70, 319-345.

Bast, T., Zhang, W.-N., and Feldon, J. (2001). The ventral hippocampus and fear conditioning in rats: different anterograde amnesias of fear after tetrodotoxin inactivation and infusion of the $\mathrm{GABA}_{\mathrm{A}}$ agonist muscimol. Experimental Brain Research, 139, 39-52.

Blanchard, R.J., and Blanchard, D.C. (2003). Bringing natural behaviors into the laboratory: a tribute to Paul MacLean. Physiology and Behavior, 79, 515-524.

Blanchard, R.J., and Blanchard, D.C. (1990). An ethoexperimental analysis of defense, fear and anxiety. In McNaughton, N., Andrews, G., editors. Anxiety, Dunedin: Otago University Press, 124-133.

Blanchard, R.J., and Blanchard, D.C. (1972a). Effects of hippocampal lesions on the rat's reaction to a cat. Journal of Comparative and Physiological Psychology, 78, 7782. 
Blanchard, D.C., and Blanchard, R.J. (1972b). Innate and conditioned defensive reactions to threat in rats with amygdaloid lesions. Journal of Comparative Physiological Psychology, 81, 281-290.

Blanchard, R.J., and Blanchard, D.C. (1969). Passive and active reactions to fear-eliciting stimuli. Journal of Comparative and Physiological Psychology, 68, 129-135.

Blanchard, R.J., Blanchard, D.C., and Fial, R.A. (1970). Hippocampal lesions in rats and their effect on activity, avoidance, and aggression. Journal of Comparative and Physiological Psychology, 71, 92-102.

Blanchard, R.J., Blanchard, D.C., and Hori, K. (1989). An ethoexperimental approach to the study of defense. In: Blanchard RJ, Brian PF, Blanchard DC, editors. Ethoexperimental approaches to the study of behavior, vol. 48. Dordrecht: Kluwer Academic Publishers, 114-136.

Blanchard, D.C., Blanchard, R.J., Lee, E.M.C., and Fukunaga, K.K. (1977). Movement arrest and the hippocampus. Physiological Psychology, 5, 331-335.

Blanchard, D.C., Blanchard, R.J., and Rogers, R.J. (1990). Pharmacological and neural control of anti-predator defense in the rat. Aggressive Behavior, 16, 165-175.

Blanchard, D.D., Li, C.I., Hubbard, D., Markham, C.M., Yang, M., Takahashi, L.T., and Blanchard, R.J. (2003). Dorsal premammillary nucleus differentially modulates defensive behaviors induced by different threat stimuli in rats. Neuroscience Letters, 345, 145-148.

Blanchard, R.J., Yang, M., Li, C-I., Gervacio, A., and Blanchard, D.C. (2001). Cue and context conditioning of defensive behaviors to cat odor stimuli. Neuroscience and Biobehavioral Reviews, 25, 587-595.

Burgess, N., Maguire, E.A., and O'Keefe, J. (2002). The human hippocampus and spatial and episodic memory. Neuron, 35, 625-641.

Canteras, N.S. (2002). The medial hypothalamic defensive system: Hodological organization and functional implications. Pharmacology, Biochemistry and Behavior, 71, 481-491.

Canteras, N.S., Ribeiro-Barbosa, E.R., and Comoli, E. (2001). Tracing from the dorsal premammillary nucleus prosencephalic systems involved in the organization of innate fear responses. Neuroscience and Biobehavioral Reviews, 25, 661-668. 
Canteras, N.S., Chiavegatto, S., Ribeiro do Valle, L.E., and Swanson, L.W. (1997). Severe reduction of defensive behavior to a predator by discrete hypothalamic chemical lesions. Brain Research Bulletin, 44, 297-305.

Canteras, N.S., Simerly, R.B., and Swanson, L.W. (1994). Organization of projections from the ventromedial nucleus of the hypothalamus: a Phaseolus vulgaris leucoagglutinin study in the rat. The Journal of Comparative Neurology, 348, 4179.

Canteras, N.S., and Swanson, L.W. (1992a). Projections of the ventral subiculum to the amygdala, septum, and hypothalamus: A PHAL anterograde tract-tracing study of the rat. Journal of Comparative Neurology, 324, 180-194.

Canteras, N.S., and Swanson, L.W. (1992b). The dorsal premammillary nucleus: an unusual component of the mammillary body. Proc National Academy of Science USA, 89, 10089-10093.

Comoli, E., Ribeiro-Barbosa, E.R., and Canteras, N.S. (2000). Afferent connections of the dorsal premammillary nucleus. The Journal of Comparative Neurology, 423, 83-98.

Deacon, R.M., Bannerman, D.M., and Rawlins, J.N. (2002). Anxiolytic effects of cytotoxic hippocampal lesions in rats. Behavioral Neuroscience, 116, 494-497.

Degroot, A., Kashluba, S., and Treit, D. (2001). Septal GABAergic and hippocampal cholinergic systems modulate anxiety in the plus-maze and shock probe tests. Pharmacology, Biochemistry and Behavior, 69, 391-399.

Degroot, A., and Treit, D. (2004). Anxiety is functionally segregated within the septohippocampal system. Brain Research, 1001, 60-71.

Degroot, A., and Treit, D. (2003) Septal Gabaergic and hippocampal cholinergic systems interact in the modulation of anxiety. Neuroscience, 117, 493-501.

Degroot, A., and Treit, D. (2002). Dorsal and ventral hippocampal cholinergic systems modulate anxiety in the plus-maze and shock-probe test. Brain Research, 949, 6070.

Dielenberg, R.A., Hunt, G.E., McGregor, I.S. (2001). 'When a rat smells a car': the distribution of fos immunoreactivity in rat brain following exposure to a predatory odor. Neuroscience, $\quad 104,1085-1097$

Dielenberg, R.A., and McGregor, I.S. (2001). Defensive behavior in rats towards predatory odors: a review. Neuroscience and Biobehavioral Reviews, 25, 597609. 
Fanselow, M.S. (2000). Contextual fear, gestalt memories, and the hippocampus. Behavioral Brain Research, 110, 73-81.

Gale, G.D., Anagnostaras, S.G., and Fanselow, M.S. (2001). Cholinergic modulation of Pavlovian fear conditioning: effects of intrahippocampal scopolamine infusion. Hippocampus, 11, 371-376.

Gray, J.A., and McNaughton, N. (2000). The Neuropsychology of anxiety, $2^{\text {nd }}$ ed. Oxford:Oxford University Press.

Jarrard, L.E. (1993). On the role of the hippocampus in learning and memory in the rat. Behavior Neural Biology, 60, 9-26.

Kim, C., Kim, C.C., Kim, J.K., Kim, S.M., Chang, H.K., Kim, J.Y., and Lee, I.G. (1971). Rear response and aggressive behavior of hippocampectomized house rats. Brain Research, 29, 237-251.

Kjelstrup, K.G., Tuvnes, F.A., Steffenach, H.A., Murison, R., Moser, E.I., and Moser, M.B. (2002). Reduced fear expression after lesions of the ventral hippocampus. $P N A S, 99,10825-10830$.

Koo, J.W., Han, J-S. and Kim, J.J. (2004). Selective neurotoxic lesions of basolateral and central nuclei of the amygdala produce differential effects on fear conditioning. The Journal of Neuroscience, 24, 7654-7662.

Li, C.I., Maglinao, T.L., and Takahashi, L.K. (2004). Medial amygdala modulation of predator odor-induced unconditioned fear in the rat. Behavioral Neuroscience, $118,324-332$.

Manns, J.R., Hopkins, R.O., and Squire, L.R. (2003) Semantic memory and the human hippocampus. Neuron, 38, 127-133.

Maren, S., Aharonov, G., and Fanselow, M.S. (1997). Neurotoxic lesions of the dorsal hippocampus and Pavlovian fear conditioning in rats. Behavioral Brain Research, $88,261-274$.

Maren, S., and Fanselow, M.S. (1997). Electrolytic lesions of the fimbria/fornix, dorsal hippocampus, or entorhinal cortex produce anterograde deficits in contextual fear conditioning in rats. Neurobiology of learning and memory, 67, 142-149.

Markham, C.M., Blanchard, D.C., Canteras, N.S., Cuyno, C.D., and Blanchard, R.J. (2004). Modulation of predatory odor processing following lesions to the dorsal premammillary nucleus. Neuroscience Letters, 372, 22-26. 
McHugh, S.B., Deacon, R.M., Rawlins, J.N.P., and Bannerman, D.M. (2004). Amygdala and ventral hippocampal lesions contribute differentially to mechanisms of fear and anxiety. Behavioral Neuroscience, 118, 63-78.

McNaughton, N., Corr, P.J. (2004). A two-dimensional neuropsychology of defense: fear/anxiety and defensive distance. Neuroscience and Biobehavioral Reviews, $28,285-305$.

McNish, K.A., Gewirtz, J.C., and Davis, M. (1997). Evidence of contextual fear after lesions of the hippocampus: a disruption of freezing but not fear-potentiated startle. The Journal of Neuroscience, 17, 9353-9360.

Menard, J., and Treit, D. (1999). Effects of centrally administered anxiolytic compounds in animal models of anxiety. Neuroscience and Biobehavioral Reviews, 23, 591613.

Menard, J., and Treit, D. (2001). The anxiolytic effects of intra-hippocampal midazolam are antagonized by intra-septal L-glutamate. Brain Research, 888, 163-166.

Matus-Amat, P., Higgin,s E.A., Barrientos, R.M., and Rudy, J.W. (2004). The role of the dorsal hippocampus in the acquisition and retrieval of context memory representations. The Journal of Neuroscience, 24, 2431-2439.

O'Keefe, J., and Nadel, L. (1978). The hippocampus as a cognitive map. Oxford: Oxford University Press.

Paxinos, G., and Watson, C. (1998). The Rat Brain in Stereotaxic Coordinates. Academic Press Sydney.

Petrovich, G.D., Risold, P.Y., and Swanson, L.W. (1996). Organization of the projections of the basomedial nucleus of the amygdala: a PHAL study in the rat. Journal of Comparative Neurology, 374, 387-420.

Petrovich, G.D., Canteras, N.S., and Swanson, L.W. (2001). Combinatorial amygdalar inputs to hippocampal domains and hypothalamic behavior systems. Brain Research Reviews, 38, 247-289.

Phillips, R.G., and LeDoux, J.E. (1992). Differential contribution of amygdala and hippocampus to cued and contextual fear conditioning. Behavioral Neuroscience, $106,274-285$.

Pikkarainen, M., Ronkko, S., Savander, V., Insausti, R., and Pitkanen, A. (1999). Projections from the lateral, basal, and accessory basal nuclei of the amygdala to the hippocampal formation in rat. The Journal of Comparative Neurology, 403, 229-260. 
Richmond, M.A., Yee, B.K., Pouzet, B., Veenman, L., Rawlins, J.N.P., Feldon, J., and Bannerman, D.M. (1999). Dissociating context and space within the hippocampus: effects of complete, dorsal, and ventral excitotoxic hippocampal lesions on conditioned freezing and spatial learning. Behavioral Neuroscience, $113,1189-1203$.

Risold, P.Y., Canteras, N.S., Swanson, L.W. (1994). Organization of projections from the anterior hypothalamic nucleus: a Phaseolus vulgaris leucoagglutinin study in the rat. Journal of Comparative Neurology, 348, 1-40.

Risold, P.Y., and Swanson, L.W. (1997). Connections of the rat lateral septal complex. Brain Research Reviews, 24, 115-195.

Risold, P.Y., and Swanson, L.W. (1996). Structural evidence for functional domains in the rat hippocampus. Science, 272, 1484-1486.

Rosen, J.B. (2004). The neurobiology of conditioned and unconditioned fear: A neurobehavioral system analysis of the amygdala.

Sacchetti, B., Lorenzini, C.A., Baldi, E., Tassoni, G., and Bucherelli, C. (1999). Auditory thalamus, dorsal hippocampus, basolateral amygdala, and perirhinal cortex role in the consolidation of conditioned freezing to context and to acoustic conditioned stimulus in the rat. The Journal of Neuroscience, 19, 9570-9578.

Sanders, M.J., Wiltgen, B.J., and Fanselow, M.S. (2003). The place of the hippocampus in fear conditioning. European Journal of Pharmacology, 463, 217-223.

Swanson, L.W. (1998). Brain maps: Structure of the rat brain, $2^{\text {nd }}$ revised ed. Amsterdam: Elsevier Science B.V.

Swanson, L.W., and Cowan, W.M. (1977). An autoradiographic study of the organization of the efferent connections of the hippocampal formation in the rat. Journal of Comparative Neurology, 172, 49-84.

Squire, L.R., Stark, C.E.L, and Clark, R.E. (2004). The medial temporal lobe. Annual Review of Neuroscience, 27, 279-306.

Squire, L.R. (1992). Memory and the hippocampus: a synthesis from findings with rats, monkeys and humans. Psychology Reviews, 99, 195-231.

Treit, D., and Menard, J. (1997). Dissociations among the anxiolytic effects of septal, hippocampal, and amygdaloid lesions. Behavioral Neuroscience, 111, 653-658. 
Trivedi, M.A., and Coover, G.D. (2004). Lesions of the ventral hippocampus, but not the dorsal hippocampus, impair conditioned fear expression and inhibitory avoidance on the elevated T-maze. Neurobiology of Learning and Memory, 81, 172-184.

Wouterlood, F.G., Saldana, E., and Witter, M.P. (1990). Projection from the nucleus reunions thalami to the hippocampal region: light and electron microscopic tracing study in the rat with the anterograde tracer Phaseolus vulgaris leucoagglutinin. Journal of Comparative Neurology, 296, 179-203.

Zhang, W.-N., Bast, T., and Feldon, J. (2001). The ventral hippocampus and fear conditioning inrats: different anterograde amnesias of fear after infusion of $\mathrm{N}$ methyl-D-aspartate or in noncompetitive antagonist MK-801 into the ventral hippocampus. Behavioral Brain Research, 126, 159-174. 